\title{
APPLICATIONS OF LUSTERNIK-SCHNIRELMANN CATEGORY AND ITS GENERALIZATIONS
}

\section{JOHN OPREA}

\section{Communicated by Vasil V. Tsanov}

\begin{abstract}
This paper explores some applications of Lusternik-Schnirelmann theory and its recent offshoots. In particular, we show how the LS category of real projective space leads to the Borsuk-Ulam theorem and the Brouwer fixed point theorem. After the development of some LS categorical tools, we also show the importance of LS category in understanding the Arnold conjecture on fixed points of Hamiltonian diffeomorphisms. We then examine ways in which LS category fits into the framework of differential geometry. In particular, we give a refinement of Bochner's theorem on the first Betti number of a non-negatively Ricci-curved space and a Bochner-like corollary to a recent theorem of Kapovitch-PetruninTuschmann. Finally, we survey the new LS categorical notion of topological complexity and its relation to the motion planning problem in robotics.
\end{abstract}

\section{Contents}

1 Introduction to LS Category $\quad \mathbf{6 0}$

1.1 Background . . . . . . . . . . . . . . . . . 60

1.2 Basic Properties of LS Category . . . . . . . . . . . . . . . 61

2 A Classical Application $\quad 63$

2.1 The Lusternik-Schnirelmann theorem . . . . . . . . . . . . . . . . 63

2.2 Antipodal Maps . . . . . . . . . . . . . . . . . . . . . . 64

2.3 The Brouwer Fixed Point Theorem . . . . . . . . . . . . . . 65

3 A Reformulation of Category and New Invariants 66

3.1 Reformulation of Category . . . . . . . . . . . . . . . . . . 66

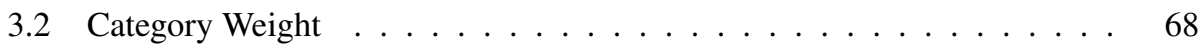

3.3 Sectional Category . . . . . . . . . . . . . . 69

4 Critical Points $\quad \mathbf{7 2}$

4.1 The $\mathrm{LS}$ Theorem . . . . . . . . . . . . . . . . . . 72

4.2 Morse Theory . . . . . . . . . . . . . . . . . . 73

4.3 LS Category in Symplectic Geometry . . . . . . . . . . . . . 75

doi: 10.7546/jgsp-36-2014-59-97 59 
5 Bochner-Type Theorems $\quad \mathbf{8 0}$

5.1 Introduction . . . . . . . . . . . . . . . 80

5.2 Non-Negative Ricci Curvature and one-Category . . . . . . . . . . . 81

5.3 Almost Non-Negative Sectional Curvature . . . . . . . . . . . . . . 81

6 Topological Complexity $\quad \mathbf{8 4}$

6.1 Introduction . . . . . . . . . . . . . . . . . . . . . . 84

6.2 Smale's Topological Complexity . . . . . . . . . . . . . . . . . 85

6.3 Farber's Topological Complexity . . . . . . . . . . . . . . . . . 88

$\begin{array}{lll}7 & \text { Final Words } & 95\end{array}$

$\begin{array}{ll}\text { References } & 95\end{array}$

\section{Introduction to LS Category}

\subsection{Background}

Definition 1. The Lusternik-Schnirelmann category of a space $X$, denoted cat $(X)$, is the smallest integer $k$ so that $X$ can be covered by $(k+1)$ open sets $U_{0}, U_{1}, \ldots$, $U_{k}$, each of which is contractible to a point in $X$. Such a covering is called a categorical covering.

LS category is an important numerical invariant in algebraic topology, critical point theory and symplectic geometry (see [4]). In this survey, which is written with nontopologists in mind, we will describe diverse ways in which LS-categorical ideas interact with other areas of Mathematics. Some of these areas are rather surprising and rather far from the original motivations of Lusternik and Schnirelmann.

In the 1920's three separate approaches arose that illuminated the relationship between the analysis and topology of smooth manifolds. In Europe, Georges de Rham showed that the cohomology of differential forms could be identified with the topologically defined singular cohomology. In the USA, Marston Morse created his "Morse theory" that re-constructed a manifold from the (non-degenerate) critical points of a smooth function on the manifold according to their indices. In Russia, Lusternik and Schnirelmann were also interested in critical points of smooth functions, but because they considered more general types than non-degenerate ones, they concentrated on relating critical point structure to a new type of homotopy invariant, now called LS category (or simply, category).

In order to describe various results associated with LS category, we will refer to certain basic notions of homotopy theory. While we cannot review all these ideas 
here, let us simply mention a few important ones. Recall that two maps $f, g: X \rightarrow$ $Y$ are homotopic if there is another map $H: X \times I \rightarrow Y$ with $H(x, 0)=f(x)$ and $H(x, 1)=g(x)$. We write $f \simeq g$ when $f$ and $g$ are homotopic. Two spaces $X$ and $Y$ have the same homotopy type, written $X \simeq Y$, if there are maps $f: X \rightarrow Y$, $g: Y \rightarrow X$ such that $f \circ g \simeq \operatorname{id}_{Y}$ and $g \circ f \simeq \operatorname{id}_{X}$. The important point is that spaces of the same homotopy type have the same algebraic invariants (homology, cohomology, homotopy groups etc.) associated with them. In particular, a space $X$ is contractible if it has the homotopy type of a point $*$. A map $f: X \rightarrow Y$ is nullhomotopic if $f \simeq *$, where $*$ refers to the constant map taking all of $X$ to a single point. Basic homotopical invariants of a space $X$ are the homotopy groups, denoted by $\pi_{k}(X)$ and defined as the homotopy classes of maps $S^{k} \rightarrow X$ (which preserve certain basepoints), where $S^{k}$ is the $k$-sphere. Of course, the most important homotopy group is $\pi_{1}(X)$, the fundamental group of $X$. Other invariants such as homology and cohomology are harder to define (but easier to compute). Now let's move on to category.

\subsection{Basic Properties of LS Category}

The first calculations of (and applications of) category use some rather simple properties that, nevertheless, require fairly sophisticated algebraic-topological notions. Since we are interested in applications, we shall list these properties here without proof. Later we shall see how to build on these properties to derive new estimates of category. (The interested reader may consult [4] for proofs.)

Properties 2. The basic properties of LS category that we shall use are the following (see [4] for instance).

1. Category is a homotopy type invariant. This means that spaces $X$ and $Y$ with $X \simeq Y$ have $\operatorname{cat}(X)=\operatorname{cat}(Y)$.

2. The cup length of a space $X$ is the largest integer $k$ such that there exists a product $x_{1} \cdots x_{k} \neq 0$, with $x_{i} \in H^{*}(X ; A)$. Here we use the fact that cohomology supports a product structure for a coefficient ring $A$. The coefficient ring $A$ may vary and the cup length may be considered for any coefficients. The fundamental relation between cup length and category is $\operatorname{cup}(X) \leqslant \operatorname{cat}(X)$

3. An upper bound for category is given by $\operatorname{cat}(X) \leqslant \operatorname{dim}(X)$ (where, for paracompact spaces more general than manifolds, $\operatorname{dim}(X)$ denotes the covering dimension of $X)$. In fact, it is possible to show that, if $\pi_{k}(X)=0$ for $0 \leqslant k \leqslant n-1$, then $\operatorname{cat}(X) \leqslant \operatorname{dim}(X) / n$. 
4. Fundamental Estimate. Combining the previous two results gives

$$
\operatorname{cup}(X) \leqslant \operatorname{cat}(X) \leqslant \frac{\operatorname{dim}(X)}{n}
$$

where $\pi_{j}(X)=0$ for $j=1, \ldots, n-1$.

Theorem 3. Let $\mathbb{R P}^{n}$ denote real projective $n$-space. Then

$$
\operatorname{cat}\left(\mathbb{R P}^{n}\right)=n \text {. }
$$

Proof: Recall that $H^{*}\left(\mathbb{R P}^{n} ; \mathbb{Z}_{2}\right)=\mathbb{Z}_{2}\left[x_{1}\right] /\left(x_{1}^{n+1}\right)$, a truncated polynomial algebra on a degree one generator $x_{1}$. Hence, $\operatorname{cup}\left(\mathbb{R P}^{n}\right)=n$ and we have, by the Fundamental Estimate, Property 2 - 4

$$
n=\operatorname{cup}\left(\mathbb{R} \mathrm{P}^{n}\right) \leqslant \operatorname{cat}\left(\mathbb{R} \mathrm{P}^{n}\right) \leqslant \operatorname{dim}\left(\mathbb{R} \mathrm{P}^{n}\right)=n
$$

so $\operatorname{cat}\left(\mathbb{R P}^{n}\right)=n$.

Example 4. Here are some simple LS category calculations (which belie the generic difficulty of computation).

1. $\operatorname{cat}\left(S^{k}\right)=1$ for any $k$. This follows because a sphere can be covered by (slightly fattened, so open) upper and lower hemispheres which are homeomorphic to disks and are thus contractible. Having two such sets means that category is equal to one. (The same proof shows that any space that is the suspension of another space has category one.)

2. $\operatorname{cat}\left(T^{k}\right)=k$. This follows because a $k$-torus is a product of $k$ circles, $T^{k}=S^{1} \times \cdots \times S^{1}$ and this means that the cohomology $H^{*}\left(T^{k} ; \mathbb{Z}\right)$ is an exterior algebra on $k$ generators. There is then a product of length $k$, so $\operatorname{cup}\left(T^{k}\right)=k$. Since $\operatorname{dim}\left(T^{k}\right)=k$ as well, the Fundamental Estimate gives the result.

3. $\operatorname{cat}\left(\mathbb{C P}^{k}\right)=k$. This follows because the cohomology of complex projective space is known to be $H^{*}\left(\mathbb{C P}^{k} ; \mathbb{Z}\right)=\mathbb{Z}\left[x_{2}\right] /\left(x_{2}^{k+1}\right)$, a truncated polynomial algebra on a degree two generator $x_{2}$. Thus, $\operatorname{cup}\left(\mathbb{C P}^{k}\right)=k$. Now, $\pi_{1}\left(\mathbb{C P}^{k}\right)=0$, so the upper bound in the Fundamental Estimate is $\operatorname{dim}\left(\mathbb{C P}^{k}\right) / 2=2 k / 2=k$, so we obtain the result.

4. The cone on $X, C X$, is obtained by taking the product $X \times I$ and crushing $X \times\{1\}$ to a point. The mapping cone of a map $f: X \rightarrow Y$ is the quotient space

$$
C f=Y \cup_{f} C X=\frac{Y \dot{\cup} C X}{(x, 0) \sim f(x)} .
$$


Extend a categorical cover of $Y$ by fattening the open sets a little past the identifications $(x, 0) \sim f(x)$ and add an open set in the cone that contracts to the cone point. This gives a categorical covering of $C f$ with one set more than the covering of $Y$. Thus, we have $\operatorname{cat}(C f) \leqslant \operatorname{cat}(Y)+1$.

\section{A Classical Application}

\subsection{The Lusternik-Schnirelmann theorem}

In this section, we will show that the calculation of the Lusternik-Schnirelmann category of real projective spaces provides a direct path to proving a version of the Borsuk-Ulam and Brouwer fixed point theorems. Lusternik and Schnirelmann proved the following result.

Theorem 5 (Lusternik-Schnirelmann Theorem) If $S^{n}$ is covered by open (or closed) sets $C_{1}, \ldots, C_{n+1}$, then at least one $C_{i}$ contains antipodal points.

Proof: Assume no $C_{i}$ contains antipodal points. Take $S^{n} \subset B^{n+1}$ and let $A_{i} \subset$ $B^{n+1}$ be the closed set defined by connecting radial segments from the origin to each point of $C_{i}$. Note that $A_{i}$ contracts to the origin.

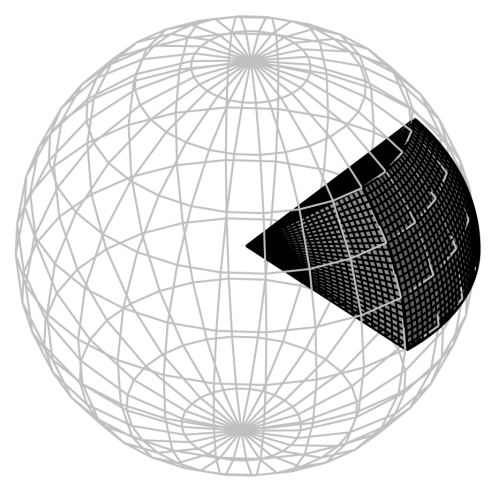

Figure 1. The radial extension $A_{i}$ of a closed set $C_{i}$.

$\mathbb{R P}^{n+1}=B^{n+1} / \sim$, where $\sim$ identifies points on the boundary $S^{n}$ with their antipodes. Note that $A_{i} \hookrightarrow \mathbb{R} \mathrm{P}^{n+1}$ is injective by the hypothesis. Also, $A_{i}$ contracts to a point in $\mathbb{R P} \mathrm{P}^{n+1}$ as well since there are no identifications on $A_{i}$. Since $\mathbb{R} \mathrm{P}^{n+1}$ is covered by $A_{1}, \ldots, A_{n+1}$, then

$$
\operatorname{cat}\left(\mathbb{R} \mathrm{P}^{n+1}\right) \leqslant n
$$


This then contradicts Theorem 3 .

Notice that we have really said that if we know that $\operatorname{cat}\left(\mathbb{R P}^{n}\right)=n$ for every $n>0$, then we know the Lusternik-Schnirelmann theorem for every $n$ as well. Surprisingly, there is a converse.

Proposition 6. If the Lusternik-Schnirelmann theorem holds for all $n>0$, then $\operatorname{cat}\left(\mathbb{R P}^{n}\right)=n$ for every $n>0$ as well.

Proof: Suppose cat $\left(\mathbb{R P}^{n}\right)=k$ for $k<n$ and let $U_{0}, \ldots, U_{k}$ be a categorical cover of $\mathbb{R P}^{n}$. Each inclusion $i_{j}: U_{j} \hookrightarrow \mathbb{R} \mathrm{P}^{n}$ is nullhomotopic, so the pullback covering is trivial: $i_{j}^{*}\left(S^{n}\right)=U_{j} \times\{0,1\} \rightarrow U_{j}$. Note that $U_{j} \times\{1\}$ is the antipodal image of $U_{j} \times\{0\}$. Let $V_{j}=U_{j} \times\{0\} \subset S^{n}$ for $j=0, \ldots, k$ and let $W=S^{n}-\cup_{j} V_{j}$. Note that $W$ is a closed (and, hence, compact) set and that each $V_{j}$ has the property that it does not contain antipodal points.

We first claim that $W$ does not contain antipodal points. Let $x \in W$. By the definition of $W$, because the $V_{j}$ and their antipodal images cover $S^{n}, x$ is in the antipodal image of some $V_{j}$. But then, $-x \in V_{j}$, so $-x \notin W$.

We need an open set $\widetilde{W}$ to add to the set of $V_{j}$, so we now claim that there exists some $\epsilon>0$ such that

$$
W_{\epsilon}=\left\{x \in S^{n} ; d(x, W)<\epsilon\right\}
$$

contains no antipodal points. Suppose not. Then we can take $\epsilon_{i} \rightarrow 0$ with $x_{i},-x_{i} \in$ $W_{\epsilon_{i}}$. There then exists a convergent subsequence $x_{i} \rightarrow y \in W$ (since $d(y, W)=$ $0)$. Now, we also have $-x_{i} \rightarrow-y$ and $d\left(-x_{i}, W\right)=0$ since taking antipodes is continuous. Hence, $-y \in W$ also and this contradicts the fact that $W$ does not contain antipodal points. Therefore, take any $\epsilon$ which gives $\widetilde{W}=W_{\epsilon}$ not containing antipodal points.

Now $\left\{V_{j}\right\}_{j=0}^{j=k} \cup \widetilde{W}$ is a cover of $S^{n}$ with $k+2 \leqslant n+1$ sets. Because none of these sets contain antipodal points, by adding empty sets if necessary, we obtain $n+1$ sets covering $S^{n}$ each of which does not contain antipodal points. This contradicts the Lusternik-Schnirelmann theorem.

\subsection{Antipodal Maps}

A map $f: S^{n} \rightarrow S^{n-1}$ is an antipodal map if $f(-x)=-f(x)$ for all $x \in S^{n}$. We have the following consequence of the Lusternik-Schnirelmann theorem.

Theorem 7. There are no antipodal maps $f: S^{n} \rightarrow S^{n-1}$. 
Proof: Suppose an antipodal map $f: S^{n} \rightarrow S^{n-1}$ exists. Represent $S^{n-1}$ as the boundary of an $n$-simplex and let the faces be denoted $F_{1}, F_{2}, \ldots, F_{n+1}$. Note that no $F_{j}$ contains antipodal points (when projected radially onto $S^{n-1}$ ). Let $G_{j}=$ $f^{-1}\left(F_{j}\right), j=1, \ldots, n+1$. The set $\left\{G_{j}\right\}$ covers $S^{n}$. Theorem 5 then says that there is some $G_{j}$ containing antipodal points, $x,-x$. Then $f(x), f(-x)=-f(x) \in F_{j}$ is a contradiction.

Theorem 7 is a version of the Borsuk-Ulam theorem and it is known that the LS theorem is, in fact, equivalent to the various versions of the Borsuk-Ulam theorem. Here we only prove the implication given in Theorem 7 since this is all we will need to prove the Brouwer fixed point theorem below. (For the interested reader, there is a generalization of these results from spheres to manifolds with involution [14].)

\subsection{The Brouwer Fixed Point Theorem}

As in almost every proof of the Brouwer theorem, we begin with the following result.

Lemma 8. If $f: D^{n} \rightarrow D^{n}$ does not have a fixed point, then there is a retraction of the disk $D^{n}$ onto its boundary $S^{n-1}$. That is, there is a map $r: D^{n} \rightarrow S^{n-1}$ such that $r \circ i_{S^{n-1}}=\mathrm{id}_{S^{n-1}}$, where $i_{S^{n-1}}: S^{n-1} \rightarrow D^{n}$ is the inclusion of the boundary.

Proof: Define a retraction by

$$
r(x)=\frac{x-f(x)}{\|x-f(x)\|} .
$$

Because $f(x) \neq x$ for all $x \in D^{n}$, the map $r$ is well-defined and continuous. Note that $r(x)=x$ if $x \in S^{n-1}$.

Theorem 9. Every map $f: D^{n} \rightarrow D^{n}$ has a fixed point.

Proof: Suppose $f$ has no fixed points and let $r: D^{n} \rightarrow S^{n-1}$ be the retraction of the lemma. Define $g: S^{n} \rightarrow S^{n-1}$ by

$$
g\left(x_{1}, \ldots, x_{n+1}\right)= \begin{cases}r\left(x_{1}, \ldots, x_{n+1}\right) & \text { if } x_{n+1} \geqslant 0 \\ -r\left(-x_{1}, \ldots,-x_{n+1}\right) & \text { if } x_{n+1} \leqslant 0\end{cases}
$$

Then $g(-x)=-g(x)$, so $g$ is antipodal. This contradicts Theorem 7 . 


\section{A Reformulation of Category and New Invariants}

We have seen that the definition of LS category and the simple cuplength-dimension estimate is sufficient to obtain classical results such as the Brouwer fixed point theorem. In order to apply category further, however, it is necessary to provide a homotopically more friendly, but more complicated equivalent definition due to $\mathrm{T}$. Ganea. For details, see [4, Chapter 1].

\subsection{Reformulation of Category}

Let $P X=\left\{\gamma: I \rightarrow X ; \gamma(0)=x_{0}\right\}$ be the contractible space of based paths with path fibration $p_{0}: P X \rightarrow X$ given by $\gamma \mapsto \gamma(1)$. We inductively construct a diagram of fibrations

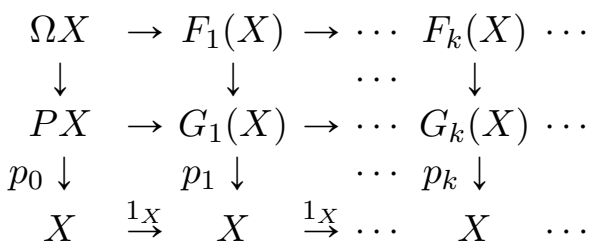

where $G_{j+1}(X)=G_{j}(X) \cup C\left(F_{j}(X)\right) \simeq G_{j}(X) / F_{j}(X)$ is the mapping cone of the previous fibre inclusion. For instance, consider the first fibration $\Omega X \rightarrow$ $P X \rightarrow X$. Take the mapping cone on the fibre inclusion $\Omega X \rightarrow P X$ to obtain $G_{1}(X)$. There is still a map to $X$ and topology has a way of making a homotopically equivalent fibration $G_{1}(X) \rightarrow X$. Iterate this process to obtain the diagram above. Through a rather long sequence of equivalences, we end up with the following characterization of LS category.

Theorem 10 (Definition-Theorem) $\operatorname{cat}(X) \leqslant n$ if and only if there is a (homotopy) section $s: X \rightarrow G_{n}(X)$. That is, in the diagram above, $p_{n} \circ s \simeq 1_{X}$.

Note that, in cohomology, we have $s^{*} \circ p_{n}^{*}=1_{H^{*}}$, so $p_{n}^{*}$ is injective. Also, it can be shown that each fibre $F_{n}(X)$ is a join of copies of the loop space $\Omega(X)$

$$
F_{n}(X)=*^{n+1} \Omega(X) .
$$

Since taking joins increases connectivity (i.e., homotopy groups vanish up to higher and higher degrees with increasing $n$ ), the long exact sequence for the homotopy groups of a fibration gives isomorphisms between the homotopy groups of $X$ and $G_{k}(X)$ through higher and higher degrees. This means that the $G_{n}(X)$ become more and more like $X$ as $n$ increases. 
Now, we need one other definition that will be important later. This is the category of a map.

Definition 11. For $f: Y \rightarrow X$, cat $(f) \leqslant n$ if and only if there is a map $s: Y \rightarrow$ $G_{n}(X)$ such that $p_{n} \circ s \simeq f$.

Properties 12. There are several properties of the reformulation diagram that will be important for us later.

1. $G_{1}(X) \simeq \Sigma \Omega(X)$, where $\Sigma Y$ denotes the suspension of the space $Y$. The suspension of $Y$ crosses the set with an interval to get $Y \times I$ and then crushes the top and bottom. (In fact, the basepoint interval must be smashed as well, but that is a technicality.) So $\Sigma Y$ looks like two cones on $Y$ attached at the $Y$ end. Now, the result follows since

$$
G_{1}(X)=G_{0}(X) \cup C(\Omega X) \simeq * \cup C(\Omega X)
$$

and attaching the cone $C(\Omega X)$ to a point crushes $\Omega X$ and gives the suspension of $\Omega(X)$.

2. If $X=K(\pi, 1)$ (i.e., all higher homotopy groups vanish, $\pi_{j}(X)=0$ for $j>1)$, then $G_{1}(X) \simeq \vee S^{1}$. This follows because $\pi_{k}(\Omega(X)) \cong \pi_{k+1}(X)$ for all $k \geqslant 0$, so $\pi_{k}(\Omega(X))=0$ for all $k \geqslant 1$ and $\pi_{0}(\Omega(X))$ is in bijection with $\pi_{1}(X)=\pi$. So, up to homotopy, $\Omega(X)$ is a set of discrete points. But then what is the suspension? The discrete set of points gives a set of disjoint intervals and these are then all smashed to a point at the top and bottom. The result, homotopically, is a set of circles all touching at a single point (i.e., a wedge of circles) $\vee S^{1}$.

3. If $X=K(\pi, 1)$, then $G_{k}(X)$ is homotopy $k$-dimensional. The proof here is much the same using the properties of the join. For instance, we have seen that $G_{1}(X) \simeq \vee S^{1}$. But $F_{1}(X)=\Omega(X) * \Omega(X)$ is the join of a discrete set of points with itself and this also is a wedge of circles. The mapping cone of $F_{1}(X) \hookrightarrow G_{1}(X)$ then attaches two-cells to $G_{1}(X)$ to obtain $G_{2}(X)$ which is then homotopy two-dimensional.

4. $\operatorname{cat}(f) \leqslant \operatorname{cat}(X)$. This is immediate from the definitions. It is also true that cat $(f) \leqslant \operatorname{cat}(Y)$ since $f$ induces $G_{k}(f): G_{k}(Y) \rightarrow G_{k}(X)$ with $p_{k}^{X} \circ$ $G_{k}(f)=f \circ p_{k}^{Y}$. 
5. If we have a commutative diagram

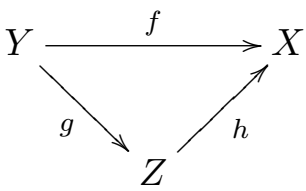

then $\operatorname{cat}(f) \leqslant \min \{\operatorname{cat}(g), \operatorname{cat}(h)\}$. This follows from Definition 11 once we note that a map $q: A \rightarrow B$ induces maps $G_{k}(A) \rightarrow G_{k}(B)$ compatible with the projections to $A$ and $B$ respectively.

\subsection{Category Weight}

One advantage of the reformulation of category is that it allows us to define new approximating invariants. Here is one that is due (independently) to Jeff Strom [25] and Yuli Rudyak [22] (building on an earlier notion of Fadell and Husseini).

Definition 13. Let $u \in H^{*}(X ; A)$, where $A$ is any coefficient group. The category weight of $u$, denoted $\operatorname{wgt}(u)$, is the maximum $k$ such that $p_{k-1}^{*}(u)=0$, where $p_{k-1}^{*}: H^{*}(X ; A) \rightarrow H^{*}\left(G_{k-1}(X) ; A\right)$ is the map induced on cohomology by $p_{k-1}: G_{k-1}(X) \rightarrow X$.

Properties 14. Here are some properties of category weight.

1. $\operatorname{wgt}(u) \leqslant \operatorname{cat}(X)$. This follows because the existence of a section $s$ of $p_{n}$ implies that $p_{n}^{*}$ is injective in cohomology.

2. $\operatorname{wgt}(u v) \geqslant \operatorname{wgt}(u)+\operatorname{wgt}(v)$. This is a harder property whose proof uses an alternative definition of category weight.

3. If $X=K(\pi, 1)$ and $u \in H^{d}(X)$, then $\operatorname{wgt}(u) \geqslant d$. Let consider the case $d=2$. We know $G_{1}(X) \simeq \Sigma \Omega X \simeq \vee S^{1}$ by Property 12 , so $p_{1}^{*}(u)=0$ since $H^{2}\left(\vee S^{1}\right)=0$. The case $d>2$ follows similarly using Property $12-3$.

4. If $f: X \rightarrow Y, u \in H^{*}(Y)$ and $f^{*}(u) \neq 0$, then $\operatorname{wgt}\left(f^{*}(u)\right) \geqslant \operatorname{wgt}(u)$. This follows by naturality: $f$ gives natural maps $G_{k}(X) \rightarrow G_{k}(Y)$ for all $k$.

5. If $f: X \rightarrow Y$ is a map and $f^{*}(u) \neq 0$, then $\operatorname{cat}(f) \geqslant \operatorname{wgt}(u)$. Look at the diagram for $\operatorname{cat}(f)=k$.

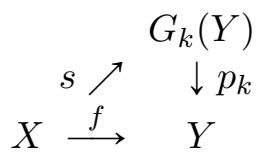


We assume that $f^{*}(u) \neq 0$, so the commutativity of the diagram then gives $p_{k}^{*}(u) \neq 0$. The definition of category weight then says that $\operatorname{wgt}(u) \leqslant k$.

\subsection{Sectional Category}

The notion of category can be extended in the following way (see [4] for instance). Recall that a fibration may be thought of as the algebraic topologist's fibre bundle.

Definition 15. Suppose $F \rightarrow E \stackrel{p}{\rightarrow} B$ is a fibration. Then the sectional category of $p$, denoted secat $(p)$, is the least integer $n$ such that there exists an open covering, $U_{0}, \ldots, U_{n}$, of $B$ and, for each $U_{i}$, a map $s_{i}: U_{i} \rightarrow E$ having $p \circ s_{i}=$ incl $_{U_{i}}$. (That is, $s_{i}$ is a local section of $p$ ).

Note that, in the reformulation fibration $\Omega(X) \rightarrow G_{0}(X)=P X \stackrel{p_{0}}{\rightarrow} X$, the total space $P X$ is contractible. Therefore, if we have an open set $U \subset X$ in the definition of sectional category, then because it factors through $P X$, the inclusion $U \hookrightarrow X$ is nullhomotopic. That is, $U$ contracts to a point in $X$. Hence, $\operatorname{cat}(X) \leqslant$ secat $\left(p_{0}\right)$. Since a basic property is that sectional category is always bounded above by the category of the base space (see (1) below), we actually have cat $(X)=$ $\operatorname{secat}\left(p_{0}\right)$. This is what we mean by extending the notion of category.

Properties 16. Consider a fibration $F \rightarrow E \stackrel{p}{\rightarrow} B$. Here are some properties of $\operatorname{secat}(p)$.

1. $\operatorname{secat}(p) \leqslant \operatorname{cat}(B)$. This follows since if $U \hookrightarrow B$ is nullhomotopic, then the Homotopy Lifting Property gives a local section of $p$ over $U$.

2. If $E$ is contractible, then $\operatorname{secat}(p)=\operatorname{cat}(B)$. (This is the case we discussed above for $G_{0}(X)$.)

3. If there are $x_{1}, \ldots, x_{k} \in \tilde{H}^{*}(B ; R)$ (any coefficient ring $R$ ) with

$$
p^{*} x_{1}=\ldots=p^{*} x_{k}=0 \text { and } x_{1} \cup \cdots \cup x_{k} \neq 0,
$$

then $\operatorname{secat}(p) \geqslant k$. This is an analogue of the cuplength estimate for ordinary category.

4. Suppose $F \stackrel{i}{\rightarrow} E \stackrel{p}{\rightarrow}$ B arises as a pullback of a fibration $\hat{p}: \widehat{E} \rightarrow \widehat{B}$ where $\widehat{E}$ is contractible (such as for a principal bundle).

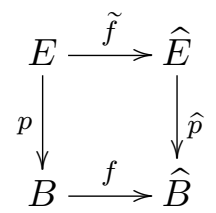


Then $\operatorname{secat}(p)=\operatorname{cat}(f)$. This says, for example, that the sectional category of principal bundles is precisely the category of the classifying map.

5. Consider the following map of fibrations

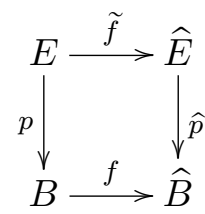

(1) If The diagram is a pullback, then $\operatorname{secat}(p) \leqslant \operatorname{secat}(\hat{p})$. This follows because an open set $\widehat{U} \subset \widehat{B}$ with a local section $\widehat{s}: \widehat{U} \rightarrow \widehat{E}$ has an open inverse image $U=f^{-1}(\widehat{U}) \subset B$ and a map $\widehat{s} \circ f: U \rightarrow \widehat{E}$ with $\hat{p} \circ \widehat{s} \circ f=f$. The pullback property then gives a map $s: U \rightarrow E$ with $p \circ s=$ incl $_{U}$.

(2) If the diagram simply commutes (i.e., is not necessarily a pullback) and $\widehat{B}=B$ with $f=\operatorname{id}_{B}$, then $\operatorname{secat}(p) \geqslant \operatorname{secat}(\hat{p})$. This follows because an open set $U \subset B$ having a local section $s: U \rightarrow E$ automatically has a local section $\widehat{f} \circ s: U \rightarrow \widehat{E}$ for $\widehat{p}$ since $\widehat{p} \circ \widehat{f}=p$.

There is a type of sectional category that will important to us later when we discuss differential geometric applications (see [19] for instance).

Definition 17. The one-category of a space $X$, denoted $\operatorname{cat}_{1}(X)$, is the least integer $n$ so that $X$ may be covered by open sets $U_{0}, \ldots, U_{n}$ having the property that, for each $U_{i}$, there is a local section $s_{i}: U_{i} \rightarrow \widetilde{X}$, where $p: \widetilde{X} \rightarrow X$ is the universal cover (so $p \circ s_{i}$ is homotopic to the inclusion $U_{i} \hookrightarrow X$ ). Thus, $\operatorname{cat}_{1}(X)=\operatorname{secat}(p: \tilde{X} \rightarrow X)$.

Before we can list properties of cat ${ }_{1}$, we need to remind the reader about a certain construction. Assume $X$ is a CW complex. That is, $X$ is inductively constructed by attaching cells in a certain allowed fashion. Now, if $\pi_{2}(X) \neq 0$, then we may attach more 3-cells to $X$ to obtain $X_{2}$ with $\pi_{2}\left(X_{2}\right)=0$. Similarly, we may attach 4-cells to obtain $X_{3}$ with $\pi_{3}\left(X_{3}\right)=0$. Continuing in this manner produces a $K(\pi, 1)$ with $\pi=\pi_{1}(X)$ and an inclusion $j_{1}: X \hookrightarrow K(\pi, 1)$ that induces an isomorphism on fundamental groups. This map $j_{1}$ classifies the universal cover of $X$, denoted $\tilde{X} \rightarrow X$, in the sense that it is the pullback over $j_{1}$ of the path fibration $P K(\pi, 1) \rightarrow K(\pi, 1)$.

Properties 18. Here are some properties of cat $_{1}$ that we will need later. 
1. $\operatorname{cat}_{1}(X)=0$ if $X$ is simply connected. This is clear from the definition or from the next property.

2. $\operatorname{cat}_{1}(X)=\operatorname{cat}\left(j_{1}: X \rightarrow K\left(\pi_{1} X, 1\right)\right)$. This follows because the universal cover $\tilde{X} \rightarrow X$ is a pullback by the classifying map $j_{1}$ of the path fibration $\left.\left.P K\left(\pi_{1} X, 1\right)\right) \rightarrow K\left(\pi_{1} X, 1\right)\right)$. Since $\left.P K\left(\pi_{1} X, 1\right)\right)$ is contractible, Property 16 (4) applies.

3. If $\pi_{1}(X)=\pi, B \pi=K(\pi, 1)$ and $k$ is the maximum degree for which $j_{1}^{*}: H^{k}(B \pi ; \mathcal{A}) \rightarrow H^{k}(X ; \mathcal{A})$ is non-trivial (for any local coefficients $\mathcal{A}$ ), then

$$
k \leqslant \operatorname{cat}_{1}(X) \leqslant \operatorname{cat}(B \pi)=\operatorname{dim}(B \pi) .
$$

Moreover, if $X=K(\pi, 1)$, then $\operatorname{cat}_{1}(X)=\operatorname{dim}(B \pi)$ (for $\left.\operatorname{dim}(B \pi)>3\right)$. This follows by Property 14.

4. Theorem (Eilenberg-Ganea). $\operatorname{cat}_{1}(X) \leqslant n$ if and only if there exists an $n$-dimensional complex $L$ such that there is a map $f: X \rightarrow L$ inducing an isomorphism

$$
f_{*}: \pi_{1}(X) \stackrel{\cong}{\rightrightarrows} \pi_{1}(L) .
$$

In one direction this follows because $\operatorname{cat}_{1}(X)=\operatorname{cat}\left(j_{1}: X \rightarrow K\left(\pi_{1} X, 1\right)\right)$ and $G_{k}\left(K\left(\pi_{1} X, 1\right)\right)$ is homotopy $k$-dimensional (see Property 12$)$. In the other direction, for an $L$ as in the condition, there is a commutative triangle

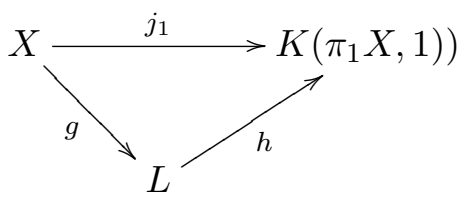

where all maps induce isomorphisms on fundamental groups. But then, by Property 12, we have

$$
\operatorname{cat}\left(j_{1}\right) \leqslant \min \{\operatorname{cat}(g), \operatorname{cat}(h)\} \leqslant \min \{\operatorname{dim}(X), \operatorname{dim}(L)\} \leqslant \operatorname{dim}(L) .
$$

Both of the next two properties are, more or less, general properties of category-like invariants (although the second does not hold for topological complexity). For cat $_{1}$, see $[18,19]$.

5. $\operatorname{cat}_{1}(X \times Y) \leqslant \operatorname{cat}_{1}(X)+\operatorname{cat}_{1}(Y)$.

6. If $p: \bar{X} \rightarrow X$ is any covering space projection, then $\operatorname{cat}_{1}(\bar{X}) \leqslant \operatorname{cat}_{1}(X)$. 
Example 19. We have $\operatorname{cat}_{1}\left(T^{n} \times X\right)=n$ if $X$ is simply connected. To see this, we use Property 18 . First, note that $j_{1}: T^{n} \times X \rightarrow T^{n}$ is the classifying map since $T^{n}=K\left(\mathbb{Z}^{n}, 1\right)$. But then, because the map on cohomology is injective, we have $n \leqslant \operatorname{cat}_{1}\left(T^{n} \times X\right)$. But we also have $\operatorname{cat}_{1}\left(T^{n} \times X\right) \leqslant \operatorname{cat}_{1}\left(T^{n}\right) \times \operatorname{cat}_{1}(X)$ and $\operatorname{cat}_{1}(X)=0$. Further, because $T^{n}=K\left(\mathbb{Z}^{n}, 1\right)$, we know $\operatorname{cat}_{1}\left(T^{n}\right)=$ $\operatorname{dim}\left(T^{n}\right)=n$. Thus, we have the result.

Example 20. We also have the interesting result that $\pi_{1}(X)$ is free if and only if $\operatorname{cat}_{1}(X)=1$. This follows from Property 18 using the fact that $K\left(\pi_{1}(X), 1\right)$ has the homotopy type of a wedge of circles (and so is homotopy one-dimensional).

Now that we have some categorical tools, let us look at various applications of category.

\section{Critical Points}

\subsection{The LS Theorem}

The original motivation of Lusternik and Schnirelmann for the introduction of category was the study of critical points of a smooth function on a manifold.

Theorem 21 (Lusternik-Schnirelmann Critical Point Theorem). Let $M$ be a smooth compact manifold and let $\operatorname{Crit}(M)$ denote the minimum number of critical points for any smooth function on $M$. Then

$$
1+\operatorname{cat}(M) \leqslant \operatorname{Crit}(M) \leqslant 1+\operatorname{dim}(M) .
$$

In fact, the second inequality is a later result of F. Takens. Furthermore, the first inequality holds in an infinite-dimensional Banach manifold context as well. See [4] for details and for the proof of the Lusternik-Schnirelmann Critical Point theorem.

Example 22. Let the torus $T^{2}$ be represented by the unit square $S q=[0,1] \times$ $[0,1]$ with $(0, y) \sim(1, y)$ and $(x, 0) \sim(x, 1)$ for all $x, y$. Define a function $G: S q \rightarrow \mathbb{R}:$

$$
G(x, y)=\sin (\pi x) \sin (\pi y) \sin (\pi(x+y)) .
$$

$G$ has 3 critical points at $(1 / 3,1 / 3),(2 / 3,2 / 3)$ and $(0,0)$, so by Theorem 21 and $\operatorname{cat}\left(T^{2}\right)=2$ we see that it is optimal. (The Hessian at $(0,0)$ is the zero matrix, so we will see below that $G$ is not a Morse function.) See Figure 2 for a graph of $G$ over Sq. Note that the corners of the square are identified to one critical point. 


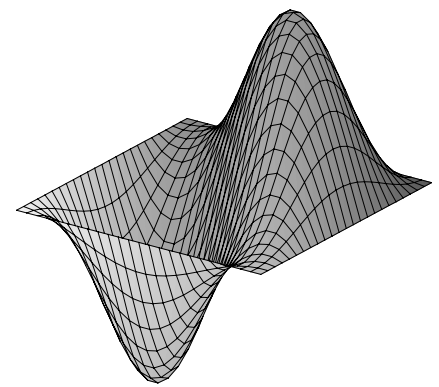

Figure 2. Plot of $G(x, y)$ on the square $S q$.

Before we leave this section, we mention a result that foreshadows the connection we shall see later between critical points and flows. (Recall that $x \in M$ is a rest point for a flow $\Psi$ if $\Psi(x, t)=x$ for all $t$.)

Theorem 23 (LS Theorem for Flows) If $X$ is a compact metric space with a gradientlike flow $\Psi$ on it and $f: X \rightarrow Y$ is a map, then

$$
1+\operatorname{cat}(f) \leqslant \operatorname{Rest}(\Psi)
$$

where $\operatorname{Rest}(\Psi)$ denotes the number of rest points of $\Psi$.

The idea is the following. If $\Psi$ were actually a gradient flow, then we would have $\dot{\Psi}(x)=\nabla f(x)$ for some function $f$, so it is clear that the critical points of $f$ correspond to points where the flow stagnates, that is, rest points of $\Psi$. It can be shown that a gradient-like flow has the same property.

At the time Theorem 21 appeared, a competing theory had been invented that also related critical points to topology. Of course, this was Morse theory. Let's review some simple aspects of it now in order to compare with LS theory.

\subsection{Morse Theory}

The main result of Morse theory is the following.

Theorem 24. If $f: M \rightarrow \mathbb{R}$ is a Morse function, then $M$ has the homotopy type of a space constructed by attaching a cell of dimension $k$ for each critical point of index $k$.

Recall that $f: M \rightarrow \mathbb{R}$ is a Morse function if, in local coordinates around each critical point, the Hessian of $f$ is invertible. The critical point is then said to be non-degenerate. Since the Hessian is a symmetric matrix, it is diagonalizable with 
real eigenvalues and the number of negative eigenvalues is the index of the critical point.

Example 25. Consider a torus $T^{2}$ with parametrization

$$
\mathbf{x}(u, v)=(r \sin (u),(R+r \cos (u)) \cos (v),(R+r \cos (u)) \sin (v)) .
$$

Define a "height function" $h: T^{2} \rightarrow \mathbb{R}$ on the torus by taking the $z$-coordinate,

$$
h(\mathbf{x}(u, v))=(R+r \cos (u)) \sin (v) .
$$

The critical points of $h(u, v)$ are at $(0, \pi / 2),(0,-\pi / 2),(\pi, 0),(\pi, \pi)$ with respective Hessian matrices

$$
\left(\begin{array}{cc}
-r & 0 \\
0 & -(R+r)
\end{array}\right), \quad\left(\begin{array}{cc}
r & 0 \\
0 & R+r
\end{array}\right), \quad\left(\begin{array}{cc}
r & 0 \\
0 & -R+r
\end{array}\right), \quad\left(\begin{array}{cc}
-r & 0 \\
0 & R-r
\end{array}\right) .
$$

These are seen in Figure 3.

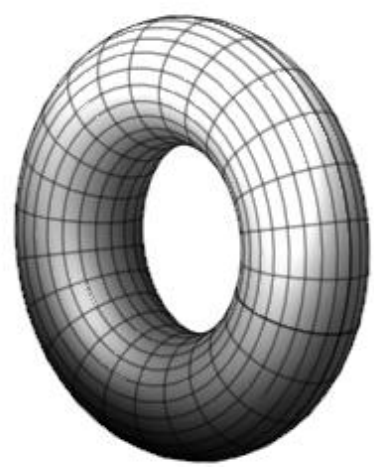

Figure 3. A vertical torus for a vertical height function.

By looking at the eigenvalues, we see that the first critical point is a maximum, the second a minimum and the last two saddles. Theorem 24 says that we begin with the minimum - a point because the index is zero. As we move up the height of the torus, we next come upon a saddle, so we attach a one-cell to the point minimum. We thus obtain a circle. The next critical point is also a saddle, so another one-cell is attached. We obtain a wedge of two circles. The maximum has index two, so we attach a two-cell to the wedge of circles that then gives the torus.

Of course, Morse theory is much more powerful than what we have intimated. In particular, by fattening the cells, we can actually assemble handles to obtain 
the homeomorphism type of the manifold. However, just from Theorem 24 we get some interesting information. The cellular homology of a cell-complex $X$ is found by taking, for each $k$, the real vector space generated by the $k$-cells, $C_{k}(X)$, and then defining a boundary homomorphism $\partial_{k}: C_{k}(X) \rightarrow C_{k-1}(X)$ with the property that $\partial_{k-1} \circ \partial_{k}=0$. Homology is then given by $H_{k}(X ; \mathbb{R})=$ $\operatorname{ker}\left(\partial_{k}\right) / \operatorname{im}\left(\partial_{k+1}\right)$. It is then clear from Theorem 24 that we have the following.

Corollary 26. If $c_{i}$ is the number of critical points of index $i$ of a Morse function, then for all $i \geqslant 0, \operatorname{dim}\left(H^{i}(M ; \mathbb{R})\right) \leqslant c_{i}$.

Example 27. From Corollary 26, we see that a Morse function on the torus $T^{2}$ has at least four critical points. This follows because we can compute homology: $\operatorname{dim}\left(H_{0}\left(T^{2} ; \mathbb{R}\right)\right)=1, \operatorname{dim}\left(H_{1}\left(T^{2} ; \mathbb{R}\right)\right)=2$, and $\operatorname{dim}\left(H_{2}\left(T^{2} ; \mathbb{R}\right)\right)=1$.

Comparing Example 22 with Example 27, we see the essential difference between the Lusternik-Schnirelmann and Morse theories and, especially, the corresponding lower bound estimates.

In Section 2 we saw how LS category impinges on fixed point theory. In this section, we have seen the consequences of LS category for critical point theory. Now we will explore a subject where LS category is the conduit for a relation between numbers of fixed and critical points.

\subsection{LS Category in Symplectic Geometry}

In Poincaré's study of celestial mechanics it became clear that a useful approach to finding closed orbits is to identify a Poincaré section and then look for periodic (or fixed) points of the associated diffeomorphism. Just before he died, Poincaré conjectured the following result.

Theorem 28 (Poincaré's Last Geometric Theorem) If $\psi: A \rightarrow A$ is an area preserving diffeomorphism of the annulus $A$ which rotates the inner and outer boundary circles in opposite directions, then $\psi$ has at least two fixed points.

Theorem 28 was proven by George Birkhoff using techniques that were very particular to dimension 2 . Note that $\operatorname{cat}(A)=1$ since $A \simeq S^{1}$, so the number of fixed points appears to be the lower bound of Theorem 21. In [1, Appendix 9], V. Arnold discussed extensions of the theorem to the torus $T^{2}$. He first noted that there are area preserving diffeomorphisms of $T^{2}$ without fixed points. For instance, translations of $\mathbb{R}^{2}$

$$
X=x+c_{1}, \quad Y=y+c_{2}
$$


descend to the quotient $T^{2}=\mathbb{R}^{2} / \mathbb{Z}^{2}$ as area preserving diffeomorphisms without fixed points. However, Arnold showed that certain hypotheses on toral diffeomorphisms allowed analogues of Theorem 28 to be proved.

Theorem 29. Suppose $\psi: T^{2} \rightarrow T^{2}$ is a diffeomorphism that satisfies the following:

- $\psi$ is homologous to the identity: that is, if we write $\psi(x, y)=(X, Y)$, then $X=x+p(x, y)$ and $Y=y+q(x, y)$ with $p, q$ periodic and $\int_{T^{2}} p=0=$ $\int_{T^{2}} q$ ( $p$ and $q$ are said to preserve the center of gravity)

- $\psi$ is area preserving: that is, $\mathrm{d} X \wedge \mathrm{d} Y=\mathrm{d} x \wedge \mathrm{d} y$

- $\psi$ is "close" to the identity.

Then $\psi$ has at least 3 (geometrically distinct) fixed points.

Proof: It can be shown from the hypotheses that there exists a periodic generating function $F(x, Y): \mathbb{R}^{2} \rightarrow \mathbb{R}$ with $p=\partial F / \partial Y, q=-\partial F / \partial x$ with $\operatorname{det} F_{x Y} \neq 0$. Note that, since $F$ is periodic, we may consider it to be a smooth function on a torus $T^{2}$. Then

$$
\psi(x, y)=\left(x+\frac{\partial F}{\partial Y}, y-\frac{\partial F}{\partial x}\right) .
$$

But now we see that the fixed points of $\psi$ correspond exactly to the critical points of $F$. By Theorem 21, we know that $F$ must have at least 3 critical points. Hence, $\psi$ has at least three fixed points.

The conditions of Theorem 29 translate into higher dimensions in the following way.

Definition 30. A manifold $\left(M^{2 n}, \omega\right)$ is symplectic if $\omega$ is a closed two-form such that $\omega^{n}$ is a volume form on $M$. (This is equivalent to saying that the form is nondegenerate.) A diffeomorphism $f: M \rightarrow M$ is a symplectomorphism if $f^{*} \omega=\omega$.

Note that, if the manifold is closed, then $\omega^{n}$ gives a non-trivial cohomology class (which we will also denote by $\omega^{n}$ ) in the top degree cohomology $H^{2 n}(M ; \mathbb{R}) \cong \mathbb{R}$. Thus, $\operatorname{cup}(M) \geqslant n$, so cat $(M) \geqslant n$. Also note that, for two-manifolds, the symplectic form and the area form are the same, so a diffeomorphism is areapreserving if and only if it is a symplectomorphism.

Example 31. The following are examples of symplectic manifolds. 
1. $\mathbb{R}^{2 n}$ with coordinates $\left(x_{1}, \ldots, x_{n}, y_{1}, \ldots, y_{n}\right)$ and $\omega=\sum_{i=1}^{n} \mathrm{~d} x_{i} \wedge \mathrm{d} y_{i}$

2. $\mathbb{C}^{n} \cong \mathbb{R}^{2 n}$

3. $T^{2 n}$, cotangent bundles $T^{*}(M)$, Kähler manifolds, orientable surfaces.

4. A nilmanifold $M=N / \pi$ is the quotient of a nilpotent Lie group $N$ by a finitely generated co-compact discrete torsionfree subgroup $\pi$. For instance the set of $3 \times 3$ upper triangular real matrices with one's on the diagonal forms a nilpotent Lie group (called the Heisenberg group) which is diffeomorphic to $\mathbb{R}^{3}$ (but with a different multiplication). A torsionfree subgroup is given by the matrices with integral entries and the resulting quotient $H$ is a closed three-manifold called the Heisenberg manifold. If $M=H \times S^{1}$, then $M$ is a four-dimensional symplectic manifold called the Kodaira-Thurston manifold. This was the first example of a symplectic non-Kähler manifold. For more on symplectic nilmanifolds, see [9] for example. Note that, since every nilpotent Lie group is diffeomorphic to some $\mathbb{R}^{n}$, then every nilmanifold is a $K(\pi, 1)$.

Symplectic geometry is the natural framework for Hamiltonian mechanics. Take a (time-dependent, say) Hamiltonian $H: M \times \mathbb{R} \rightarrow \mathbb{R}$. In coordinate-free language, each function $H_{t}$ has an associated vector field $X_{t}$ with $i_{X_{t}} \omega=\mathrm{d} H_{t}$. The timedependent vector field $X_{t}$ has integral curves (in local coordinates) $(q(t), p(t)$ ) with associated flow $\Phi: M \times \mathbb{R} \rightarrow M ; \Phi((q, p), t)=(q(t), p(t))$, where we have $(q(0), p(0))=(q, p)$. The equality $i_{X_{t}} \omega=\mathrm{d} H_{t}$ gives Hamilton's Equations for the flow

$$
\dot{q}_{i}(t)=\frac{\partial H(t, \Phi(t))}{\partial p_{i}}, \quad \dot{p}_{i}(t)=-\frac{\partial H(t, \Phi(t))}{\partial q_{i}} .
$$

Definition 32. The time-one map $\phi=\Phi_{1}=\Phi(-, 1)$ of the flow determined by a time-dependent Hamiltonian is called a Hamiltonian diffeomorphism.

Hamiltonian diffeomorphisms are the generalization of toral diffeomorphisms that preserve the center of gravity. An important characterization of Hamiltonian diffeomorphisms is that they precisely form the commutator subgroup of the group of symplectomorphisms. The generalization of Theorem 29 is now the following (see [1, Appendix 9]).

Conjecture 33 (The Arnold Conjecture). If $\phi: M \rightarrow M$ is a Hamiltonian diffeomorphism on a closed symplectic manifold $(M, \omega)$ and $\operatorname{Fix}(\phi)$ stands for the number of fixed points of $\phi$, then

$$
\operatorname{Fix}(\phi) \geqslant \operatorname{Crit}(M) .
$$


In the Conjecture, the Hamiltonian diffeomorphism can be replaced by a oneperiodic flow and the fixed points can be replaced by the one-periodic solutions of the associated Hamilton equations. See [12] for details. These are critical points of the following action functional on (contractible) loops u in M (i.e., contractible $\left.u: S^{1} \rightarrow M\right):$

$$
A_{H}(u)=-\int_{D^{2}} \tilde{u}^{*} \omega-\int_{S^{1}} H_{t}(u(t)) \mathrm{d} t
$$

where $\widetilde{u}: D^{2} \rightarrow M$ is an extension to $D^{2}$ of the loop $u: S^{1} \rightarrow M$ using $u$ 's contractibility. The important thing to notice is that the functional is not well defined! Different extensions to the disk can give different results - unless $\left.\omega\right|_{\pi_{2}(M)}=0$ - and this was Floer's approach to the problem. Geometrically, this condition is saying that

$$
\int_{S^{2}} g^{*} \omega=0
$$

for all $g: S^{2} \rightarrow M$. Pasting $\widetilde{u}$ and $\widehat{u}$ together along $S^{1}$ gives a map $g: S^{2} \rightarrow M$ with

$$
0=\int_{S^{2}} g^{*} \omega=\int_{D^{2}} \widetilde{u}^{*} \omega-\int_{D^{2}} \widehat{u}^{*} \omega
$$

showing that $A_{H}$ is then well-defined. So we restrict ourselves to symplectic manifolds $(M, \omega)$ with $\left.\omega\right|_{\pi_{2}(M)}=0$ and call these symplectically aspherical manifolds. Floer was able to then prove a weakened version of the Arnold Conjecture.

Theorem 34. If $\phi: M \rightarrow M$ is a Hamiltonian diffeomorphism on a symplectically aspherical manifold, then

$$
\operatorname{Fix}(\phi) \geqslant 1+\operatorname{cup}_{\mathbb{Z}_{2}}(M) .
$$

We say that this result is a weakened form of Conjecture 33 by virtue of Property 2 and Theorem 21. Since Floer's work there have been several proofs of the weakened conjecture for general symplectic manifolds (see for example, $[8,13]$ ). Moreover, in the spirit of Morse theory, the result can be strengthened to having a lower bound equal to the sum of the Betti numbers when the fixed points are non-degenerate.

Now, here is how LS category enters the picture. It relies on a homotopy interpretation of the condition $\left.\omega\right|_{\pi_{2}(M)}=0$. We have $H^{2}(M ; \mathbb{R}) \cong \operatorname{Hom}\left(H_{2}(M), \mathbb{R}\right)$, so think of $\omega$ as a homomorphism $\omega: H_{2}(M) \rightarrow \mathbb{R}$. The Hurewicz homomorphism (in degree 2), $h: \pi_{2}(M) \rightarrow H_{2}(M)$, is defined by $h(\alpha)=\alpha_{*}(\iota)$, where $\alpha: S^{2} \rightarrow$ $M$ is a representative of the homotopy class $\alpha \in \pi_{2}(M)$ and $\iota \in H_{2}\left(S^{2}\right) \cong \mathbb{Z}$ is a chosen (and fixed) generator. The image of the Hurewicz homomorphism, $\operatorname{im}(h)$, is then a subgroup of $H_{2}(M)$. The notation $\left.\omega\right|_{\pi_{2}(M)}=0$ then means that $\omega: H_{2}(M) \rightarrow \mathbb{R}$ vanishes on $\operatorname{im}(h) \subseteq H_{2}(M)$. Recall the following. 
Theorem 35 (Hopf's Theorem) . The universal cover classifying map $f: M \rightarrow$ $K\left(\pi_{1}(M), 1\right)$ induces isomorphisms with integral coefficients

$$
\begin{aligned}
& H_{1}(M ; \mathbb{Z}) \cong H_{1}\left(K\left(\pi_{1}(M), 1\right) ; \mathbb{Z}\right) \\
& \frac{H_{2}(M ; \mathbb{Z})}{\operatorname{im}\left(h_{2}\right)} \cong H_{2}\left(K\left(\pi_{1}(M), 1\right) ; \mathbb{Z}\right) .
\end{aligned}
$$

The second isomorphism has the following immediate implication.

Corollary 36. The condition $\left.\omega\right|_{\pi_{2}(M)}=0$ holds if and only if there exists $\omega_{\pi} \in$ $H^{2}\left(K\left(\pi_{1}(M), 1\right) ; \mathbb{R}\right)$ with $f^{*} \omega_{\pi}=\omega$ where $f: M \rightarrow K\left(\pi_{1}(M), 1\right)$ is as above.

By our results on category weight, Corollary $36 \operatorname{implies}$ that $\operatorname{wgt}(\omega)=2$ and, consequently, $\operatorname{wgt}\left(\omega^{n}\right)=2 n=\operatorname{dim}(M)$. This then says, in particular, that $\operatorname{cat}(M)=2 n=\operatorname{dim}(M)$ for a symplectically aspherical manifold. We will see what this implies below. The key link between the analysis of the Arnold Conjecture and LS category is the following result [12].

Theorem 37. Suppose $\left(M^{2 n}, \omega\right)$ is a symplectically aspherical manifold and $H: M \times$ $\mathbb{R} \rightarrow \mathbb{R}$ is a one-periodic time-dependent Hamiltonian. Then

1. There is a gradient-like flow $\Psi$ on a compact metric space $X_{\infty}$ such that

$$
\begin{array}{r}
\operatorname{Rest}(\Psi) \leqslant \text { Number of contractible one }- \text { periodic } \\
\text { orbits of the flow } \Phi \text { associated to } H \text {. }
\end{array}
$$

2. There is a map $\tau: X_{\infty} \rightarrow M$ that induces an injection in cohomology (with any coefficients $R$ )

$$
\tau^{*}: H^{*}(M ; R) \rightarrow H^{*}\left(X_{\infty} ; R\right)
$$

Recalling that we replaced fixed points by one-periodic orbits and using Theorem 37 , we have

$$
\begin{aligned}
\operatorname{Fix}(\phi) & \geqslant \text { Number of contractible one }- \text { periodic orbits } \\
& \geqslant \operatorname{Rest}(\Psi) \\
& \geqslant 1+\operatorname{cat}(\tau), \text { by Theorem } 23 .
\end{aligned}
$$

Also, since $\tau^{*}\left(\omega^{n}\right) \neq 0$ and $2 n+1=\operatorname{dim}(M)+1$, we have by Property 14 ,

$$
2 n+1 \geqslant \operatorname{Crit}(M) \geqslant \operatorname{cat}(M)+1 \geqslant \operatorname{cat}(\tau)+1 \geqslant \operatorname{wgt}\left(\omega^{n}\right)+1=2 n+1
$$

since $\omega=f^{*}\left(\omega_{\pi}\right)$ for $f: M \rightarrow K\left(\pi_{1}(M), 1\right)$. Hence, $\operatorname{Crit}(M)=\operatorname{cat}(\tau)+1$. This then leads to a proof of the following original version of Arnold's Conjecture. 
Theorem $38([21,23])$. Let $(M, \omega)$ be a closed symplectically aspherical manifold and let $\operatorname{Crit}(M)$ denote the minimum number of critical points for any smooth function $f: M \rightarrow \mathbb{R}$. If $\phi: M \rightarrow M$ is a Hamiltonian diffeomorphism and $\operatorname{Fix}(\phi)$ stands for the number of fixed points of $\phi$, then

$$
\operatorname{Fix}(\phi) \geqslant \operatorname{Crit}(M) \text {. }
$$

\section{Proof:}

$$
\begin{aligned}
\operatorname{Fix}(\phi) & \geqslant \text { Number of contractible one }- \text { periodic orbits } \\
& \geqslant \operatorname{Rest}(\Psi) \\
& \geqslant 1+\operatorname{cat}(\tau)=\operatorname{Crit}(M) .
\end{aligned}
$$

\section{Bochner-Type Theorems}

\subsection{Introduction}

In the 1920's, the development of algebraic topology by Alexander and Lefschetz was accompanied by the efforts of Morse, de Rham and Lusternik-Schnirelmann to relate algebraic topological invariants to global analysis. Of course, for some manifolds such as Kähler manifolds, analysis and geometry are strongly bound together and this is reflected algebraic topologically in the fact that the cohomology of a Kähler manifold satisfies the Hard Lefschetz property (see [27] for example). Another result that exemplifies this mixture of geometry, analysis and topology is the following result of S. Bochner (which on the face of it does not appear analytic!) [30].

Theorem 39. If $M$ has non-negative Ricci curvature, then $b_{1}(M) \leqslant \operatorname{dim}(M)$. Moreover, equality holds if and only if $M \cong T^{n}$ (where the torus is flat).

While the actual definition of Ricci curvature is not important for understanding the statement, note that this result is proved by relating the Hodge Laplacian to the connection Laplacian and the Ricci curvature form via the analysis associated to the so-called Weitzenböck formula (see [20] for instance). The question for us is, how can LS category impinge on this type of result? As we saw with the Arnold conjecture, LS category can be used in conjunction with an intermediary theorem that brings analysis closer to topology. Here that type of result is the following [2]. 
Theorem 40 (Cheeger-Gromoll Theorem). If $M$ has non-negative Ricci curvature, then $M$ has a finite cover $\bar{M} \cong T^{k} \times N$ where $N$ is one-connected.

It can be seen that the Cheeger-Gromoll theorem implies Bochner's theorem, but in fact, it gives much more.

Theorem 41 ([16]). If $M$ has non-negative Ricci curvature, then $b_{1}(M) \leqslant \operatorname{cat}(M)$. Moreover, equality holds if and only if $M \cong T^{n}$ (where the torus is flat).

We won't prove this theorem because we will supercede it using our extra knowledge about cat ${ }_{1}$. In fact, the second part of the statement is somewhat unsatisfactory because equality only holds in a "trivial" case. This often means that an estimate is flabby. Let's now try to improve the estimate.

\subsection{Non-Negative Ricci Curvature and one-Category}

Theorem 41 can be refined using what we know about cat 1 (see [19]).

Theorem 42. If $M$ has non-negative Ricci curvature, then $b_{1}(M) \leqslant \operatorname{cat}_{1}(M)$.

Proof: By Theorem 40, there is a splitting $\bar{M} \cong T^{k} \times N$ of a finite cover $\bar{M} \rightarrow M$. Now, we know that $b_{1}(M) \leqslant b_{1}(\bar{M})=b_{1}\left(T^{k}\right)=k$ by the transfer for finite covers. (Recall that, for a finite cover with $n$-sheets $p: \bar{X} \rightarrow X$, there is a transfer homomorphism $\tau: H^{*}(\bar{X} ; \mathbb{Q}) \rightarrow H^{*}(X ; \mathbb{Q})$ such that $\tau \circ p^{*}=n \cdot$ id.) Now, by Property 18, we have

$$
b_{1}(M) \leqslant k=\operatorname{cat}_{1}\left(T^{k} \times N\right)=\operatorname{cat}_{1}(\bar{M}) \leqslant \operatorname{cat}_{1}(M) .
$$

Example 43. $M=T^{2} \times S^{2}$ has a (product) metric with non-negative Ricci curvature. Clearly, $b_{1}(M)=2$ and by Example 19, we see that $\operatorname{cat}_{1}(M)=2$ as well. Note however, that $M$ is not a torus. Therefore, qualitatively, the estimate of Theorem 42 is much better than those found in Theorem 39 and Theorem 41.

\subsection{Almost Non-Negative Sectional Curvature}

Now let us see how this general approach can be applied to other problems on the border between geometry and topology. Because the following curvature notion is not so widely known, we give the definition. 
Definition 44. A closed smooth manifold $M^{m}$ is said to be almost non-negatively (sectionally) curved (or ANSC) if it admits a sequence of Riemannian metrics $\left\{g_{n}\right\}_{n \in \mathrm{N}}$ whose sectional curvatures and diameters satisfy

$$
\sec \left(M, g_{n}\right) \geqslant-\frac{1}{n} \quad \text { and } \quad \operatorname{diam}\left(M, g_{n}\right) \leqslant \frac{1}{n} .
$$

Here is an old result of Yamaguchi [29] about these types of manifolds.

Theorem 45 (Yamaguchi's Theorem). If $M^{m}$ is an ANSC manifold, then:

1. A finite cover of $M$ is the total space of a fibration over a torus of dimension $b_{1}(M)$

2. If $b_{1}(M)=m$, then $M^{m}$ is diffeomorphic to $T^{b_{1}(M)}$.

More recently, a beautiful structure theorem has been proved about ANSC manifolds [17].

Theorem 46 (Kapovitch-Petrunin-Tuschmann Theorem). If $M$ is an ANSC manifold, then there is a finite cover $\bar{M}$ that is the total space of a fibre bundle

$$
F \rightarrow \bar{M} \stackrel{p}{\rightarrow} N
$$

where $N=K(\pi, 1)$ is a nilmanifold (see Example 31 - 4) and $F$ is a simply connected closed manifold which is almost non-negatively curved in a generalized sense.

A fundamental question is, what is the relationship between Yamaguchi's old result and this new structure theorem of Kapovitch-Petrunin-Tuschmann. Let's begin to answer this question by first proving the following Bochner-type theorem [19].

Theorem 47. Suppose $M$ is an ANSC manifold with associated finite cover $\bar{M}$ and KPT-fibre bundle

$$
F \rightarrow \bar{M} \stackrel{p}{\rightarrow} N
$$

where $N=K(\pi, 1)$ is a nilmanifold and $F$ is a simply connected closed manifold. Then:

i) $b_{1}(M) \leqslant \operatorname{dim}(N) \leqslant \operatorname{dim}(\bar{M})=\operatorname{dim}(M)$

ii) Moreover, if the universal cover $\widetilde{M}$ has non-zero Euler characteristic, then $b_{1}(M) \leqslant \operatorname{dim}(N) \leqslant \operatorname{cat}_{1}(M)$. 
Proof: We know $b_{1}(M) \leqslant b_{1}(\bar{M})$ from the transfer. But the long exact homotopy sequence of the bundle also gives $\pi_{1}(\bar{M}) \cong \pi_{1}(N)=\pi$. By Hopf's Theorem, we know that the first homology depends only on the fundamental group, so we obtain $H_{1}(\bar{M} ; \mathbb{Q}) \cong H_{1}(\pi ; \mathbb{Q}) \cong H_{1}(N ; \mathbb{Q})$. Hence, $b_{1}(M) \leqslant b_{1}(\bar{M})=b_{1}(N)$.

Now we must invoke some results from Rational Homotopy Theory (see $[9,27]$ ). Since $N$ is a nilmanifold, it has a (rational-homotopy-theoretic) minimal model $\left(\wedge\left(x_{1}, x_{2}, \ldots, x_{k}\right), d\right)$, where each generator has degree $\left(x_{j}\right)=1$ and $k$ is the rank of the torsionfree nilpotent group $\pi$. By the general theory, the differential $d$ is zero on $x_{1}, \ldots, x_{s}$ for some $2 \leqslant s \leqslant k$ and $k=\operatorname{dim}(N)$. (The case $s=k$ is a torus.) Then $b_{1}(N)=s \leqslant k=\operatorname{dim}(N)$. Since $F \rightarrow \bar{M} \stackrel{p}{\rightarrow} N$ is a bundle, we see that $\operatorname{dim}(N) \leqslant \operatorname{dim}(\bar{M})=\operatorname{dim}(M)$. This proves i).

For ii), because $F \simeq \widetilde{M}$ and $\chi(\widetilde{M}) \neq 0$, the bundle $F \rightarrow \bar{M} \stackrel{p}{\rightarrow} N$ has a BeckerGottlieb transfer map $\tau: H^{*}(\bar{M} ; \mathbb{Z}) \rightarrow H^{*}(N ; \mathbb{Z})$ with $\tau \circ p^{*}(\alpha)=\chi(F) \cdot \alpha$, for all $\alpha \in H^{*}(N ; \mathbb{Z})$. This implies that

$$
p^{*}: H^{*}(N ; \mathbb{Q})=H^{*}(K(\pi, 1) ; \mathbb{Q}) \rightarrow H^{*}(\bar{M} ; \mathbb{Q})
$$

is injective on rational cohomology. Hence, since $\bar{M} \rightarrow N=K(\pi, 1)$ may be considered as the classifying map of the universal cover, Property 18 implies that $\operatorname{dim}(N) \leqslant \operatorname{cat}_{1}(\bar{M})$. Thus

$$
b_{1}(M) \leqslant b_{1}(N) \leqslant \operatorname{dim}(N) \leqslant \operatorname{cat}_{1}(M) .
$$

Using Theorem 47, we can see how (a topological version of) Theorem 45 follows from Theorem 46.

Theorem 48 ([19]). Suppose a closed manifold $M$ has a finite cover $\bar{M}$ that is the total space of a fibre bundle

$$
F \rightarrow \bar{M} \stackrel{p}{\rightarrow} N
$$

where $N=K(\pi, 1)$ is a nilmanifold and $F$ is a simply connected closed manifold. Then:

1. A finite cover of $M$ is the total space of a fibration over a torus of dimension $b_{1}(M)$

2. If $b_{1}(M)=m=\operatorname{dim}(M)$, then $M^{m}$ is homeomorphic to $T^{b_{1}(M)}$.

Note that we only get a homeomorphism in (2) instead of Yamaguchi's diffeomorphism. Yamaguchi uses surgery techniques to move from a homeomorphism to a diffeomorphism that are beyond the scope of this survey. 
Proof: Consider $F \rightarrow \bar{M} \stackrel{p}{\rightarrow} N$. From Theorem 47, we know that

$$
b_{1}(M) \leqslant b_{1}(\bar{M})=b_{1}(N) \leqslant \operatorname{dim}(N) \leqslant \operatorname{dim}(\bar{M})=\operatorname{dim}(M) .
$$

Now, the general construction of the nilmanifold $N$ via iterated principal $S^{1}$ bundles shows that we may start the iteration by a bundle over $T^{b_{1}(\bar{M})}$ or any torus of lower dimension. Thus, item 1 follows since a composition of fibrations is a fibration.

Now assume $b_{1}(M)=m=\operatorname{dim}(M)$. Then by the string of inequalities above, $\operatorname{dim}(N)=m=\operatorname{dim}(\bar{M})$. Because we have a fibre bundle, we see that $\operatorname{dim}(F)=$ 0 and (since $F$ is connected) we have $\bar{M}=N$. But then we have $b_{1}(N)=m=$ $\operatorname{dim}(N)$ by the inequalities. For a nilmanifold, this can only happen if $N$ is a torus $T^{m}$ and $\pi \cong \mathbb{Z}^{m}$. Now, $\bar{M}=T^{m}$ covers $M$, so $M$ is a $K(G, 1)$ where $G=\pi_{1}(M)$. Since $M$ is a closed $m$-manifold that is also a $K(G, 1)$, we have that $G$ is torsionfree. Now, $\pi \cong \mathbb{Z}^{m}$ has finite index in $G$ and $b_{1}(\pi)=m=b_{1}(M)=$ $b_{1}(G)$, so $G \cong \mathbb{Z}^{m}$ by the following.

Lemma 49. If $\pi \cong \mathbb{Z}^{m}$ is a finite index subgroup of a torsionfree group $G$ and $b_{1}(G)=m$, then $G \cong \mathbb{Z}^{m}$.

See [19] for a proof of this group-theoretic result. Hence $M=K\left(\mathbb{Z}^{m}, 1\right)$ is a homotopy torus. Since the (surgery) topological structure set for the torus is trivial, we have that $M$ is then homeomorphic to $T^{m}$.

\section{Topological Complexity}

\subsection{Introduction}

The phrase "topological complexity" has both an old and new meaning within the framework of LS category theory. The old meaning refers to Smale's measure of the complexity of an algorithm [24]. The new meaning refers to Farber's measure of the insolubility of the motion planning problem in robotics [5-7]. The interesting thing about these apparently unrelated problems is that they both rely on sectional category as the basis of their defining measures. In this section, we will present these notions with an emphasis on the categorical aspects. For properties of sectional category, see Subsection 3.3. 


\subsection{Smale's Topological Complexity}

What is an algorithm? Without having a specific definition, it would be impossible to measure complexity. Smale took a simple definition of algorithm which surprisingly fits many situations.

Definition 50. An algorithm tree is a connected directed graph $G$ with vertices $\left\{R, V_{1}, \ldots, V_{N}, L_{1}, \ldots, L_{m}\right\}$ satisfying the following conditions.

1. There are no loops, i.e., $G$ is a tree.

2. The root $R$ has only one edge and that edge comes out of $R$.

3. Each $V_{i}$ has one edge coming into it and either one or two edges coming out of it. Those $V_{i}$ with one edge coming out are called computation vertices and those with two edges coming out are called branch vertices.

4. Each leaf $L_{j}$ has only one edge coming into it.

Example 51. Here is a typical algorithm tree. Note that the algorithm proceeds from the root (where the input is made) to one of the leaves (where the output appears).

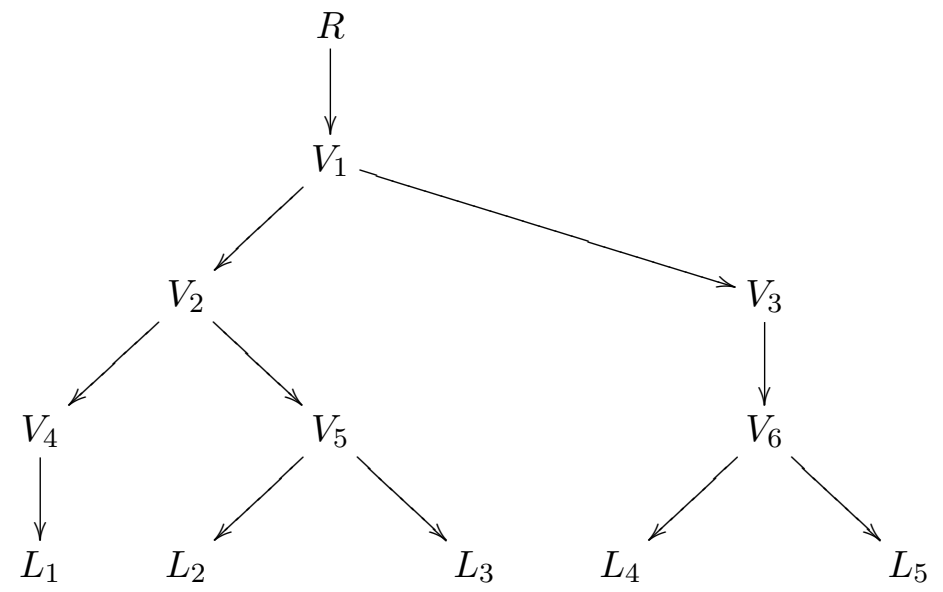

Definition 52. The Smale topological complexity of an algorithm tree $G, \tau(G)$, is the number of branch vertices in $G$. This is also equal to one fewer than the number of leaves in the tree

$$
\#\left\{L_{j}\right\}-1=\#\left\{\text { Branch } V_{i}\right\}=\tau(G) .
$$


The Smale topological complexity of a particular problem $P$ is the minimum of the topological complexities of all algorithms which solve the problem

$$
\tau(P)=\min \{\tau(G) ; G=\text { algorithm tree for } P\} .
$$

Smale's main example (which motivates the definition in fact) is to the root finding problem.

Problem 53 (Root Finding Problem) Find the roots of $P(x)$, where

$$
P(x)=a_{0}+a_{1} x+a_{2} x^{2}+\cdots+a_{d-1} x^{d-1}+x^{d} .
$$

is a monic polynomial of degree $d$ with complex coefficients. Here, the word "find" is taken to mean "find to within a given accuracy $\epsilon$ ". This problem is denoted by $R F(d, \epsilon)$.

After Smale's work, the following result was proved that gave an upper bound for the Smale topological complexity of the root finding problem.

Theorem 54 (Vassiliev) There exists an algorithm tree of topological complexity $d-1$ for the problem of determining roots of degree $d$ monic polynomials to within a given $\epsilon>0$. Thus, the topological complexity of the problem is at most $d-1$

$$
\tau(R F(d, \epsilon)) \leqslant d-1 .
$$

Let $\mathcal{P}_{d}$ denote the set of degree $d$ monic polynomials with complex coefficients. Take the mapping $\pi: \mathcal{C}^{d} \rightarrow \mathcal{P}_{d}$ given by

$$
\pi\left(\xi_{1}, \ldots, \xi_{d}\right)=\prod_{i=1}^{d}\left(x-\xi_{i}\right) .
$$

Let

$$
\begin{aligned}
\Delta & =\left\{\left(\xi_{1}, \ldots, \xi_{d}\right) ; \xi_{i}=\xi_{j} \text { for some } i \neq j\right\} \subseteq \mathcal{C}^{d} \\
\pi(\Delta) & =\Sigma=\{\text { Polynomials with repeated roots }\} \subseteq \mathcal{P}_{d} .
\end{aligned}
$$

Denote the restriction

$$
\left.\pi\right|_{\mathcal{C}^{d}-\Delta}: \mathcal{C}^{d}-\Delta \rightarrow \mathcal{P}_{d}-\Sigma
$$

by $\pi$ as well. The symmetric group on $d$ letters, $S(d)$, acts by permuting the $d$ coordinates $\xi_{i}$ and this action has no fixed points on $\mathcal{C}^{d}-\Delta$. Therefore, $\pi$ is a $(d$ !)-fold covering map. Here is Smale's result. 
Theorem 55 (Smale). For any $d$ there exists $\epsilon_{d}>0$ such that, for $\epsilon<\epsilon_{d}$, the topological complexity $\tau(R F(d, \epsilon))$ for the problem of finding roots of degree $d$ monic polynomials to within $\epsilon$ is at least the sectional category of the covering $\pi: \mathcal{C}^{d}-\Delta \rightarrow \mathcal{P}_{d}-\Sigma$. That is

$$
\tau(R F(d, \epsilon)) \geqslant \operatorname{secat}(\pi) .
$$

Proof (Sketch of Proof): It can be shown that the problem can be reduced to the case of polynomials with no repeated roots. Suppose there is an algorithm tree which solves the root finding problem. Let the solution algorithm tree be $G$ with vertices $\left\{R, V_{1}, \ldots, V_{N}, L_{1}, \ldots, L_{m}\right\}$. For $i=1, \ldots, m$, define $Z_{i}$ to be the set

$$
\begin{gathered}
\left\{P(x) \in \mathcal{P}_{d}-\Sigma ;\right. \\
\text { the output of the algorithm tree } G \text { applied to } P(x) \\
\text { exits the tree through leaf } \left.L_{i}\right\} .
\end{gathered}
$$

Since an algorithm exists we define an input-output map $\phi: \mathcal{P}_{d}-\Sigma \rightarrow \mathcal{C}^{d}$ by

$$
\phi(P(x))=\left(z_{1}, z_{2}, \ldots, z_{d}\right)
$$

(the output of the algorithm) where each $z_{i}$ satisfies $\left|z_{i}-\xi_{i}\right|<\epsilon$, for $\xi_{i}$ the true roots of $P(x)$. The branch inequalities say that $Z_{i}$ is a semi-algebraic set, so the Tietze extension theorem gives an open set $U_{i}$ containing $Z_{i}$ and an extension $\phi: U_{i} \rightarrow \mathcal{C}^{d}$. These input-output maps are "sections to within $\epsilon$ ". It requires a clever argument, but in fact these $\phi$ 's can be deformed to actual sections. But this is exactly sectional category! Therefore, we obtain

$$
\operatorname{secat}(\pi) \leqslant m-1=\#(\text { branches })=\tau(R F(d, \epsilon)) .
$$

We now see that, combined with Vassiliev's result, we have

$$
d-1 \geqslant \tau(R F(d, \epsilon)) \geqslant \operatorname{secat}(\pi) .
$$

Now, we know something about secat $(\pi)$ from Property 16 . The covering map $\pi: \mathbb{C}^{d}-\Delta \rightarrow \mathcal{P}_{d}-\Sigma$ is a principal bundle induced by a classifying map $f: \mathcal{P}_{d}-$ $\Sigma \rightarrow K(S(d), 1)$. Then, we have $\operatorname{secat}(\pi)=\operatorname{cat}(f)$. Of course, we also know how to estimate cat $(f)$ using category weight by Property 14 . For this, we need to know the highest degree where the induced map on cohomology $f^{*}$ is non-zero. For this, we recognize that in fact, $\mathcal{C}^{d}-\Delta=\mathbb{R}^{2}[d]$ is the ordered configuration space of $d$ points in $\mathbb{R}^{2}$, a space that has been studied extensively. (Here and below we use Vassiliev's notation, see [28].) The symmetric group $S(d)$ acts freely on $\mathbb{R}^{2}[d]$ by permuting the ordering and the resulting quotient is the $d$ th unordered configuration space, denoted $\mathbb{R}^{2}(d)$, which is $P_{d}-\Sigma$. 
Now, it is known that $\mathbb{R}^{2}(d)=K(B r(d), 1)$, where $B r(d)$ is the braid group and there is a homomorphism $B r(d) \rightarrow S(d)$ arising from the classifying map $f: \mathbb{R}^{2}(d) \rightarrow K(S(d), 1)$ of the covering projection $\mathbb{R}^{2}[d] \rightarrow \mathbb{R}^{2}(d)$. But here is a result about this situation that was discovered (essentially) before Smale's topological complexity was even invented.

\section{Theorem 56 (Arnold-Fuchs-Vassiliev) .}

$$
H^{d-1}(K(B r(d), 1) ; \pm \mathbb{Z})= \begin{cases}0 & \text { if } d \neq p^{q}, \text { for } p \text { prime } \\ \mathcal{Z}_{p} & \text { if } d=p^{q}, \text { for } p \text { prime }\end{cases}
$$

Moreover, the homomorphism $f^{*}: H^{*}(K(S(d), 1) ; \pm \mathbb{Z}) \rightarrow H^{*}(K(B r(d), 1) ; \pm \mathbb{Z})$ is surjective.

First, note that the notation $\pm \mathbb{Z}$ means that cohomology is taken with respect to local coefficients given by the $S(d)$-module $\mathbb{Z}$ defined by multiplying an integer by \pm 1 according to the parity of the permutation. But the properties of category weight hold for any coefficients, so because $f^{*}$ is surjective, it is simply the highest degree of $H^{*}(K(B r(d), 1) ; \pm \mathbb{Z})$. This then bounds cat $(f)$ from below. We then obtain the following from Theorem 56.

Corollary 57. For d equal to a power of some prime, there exists $\epsilon_{d}>0$ such that, for $\epsilon<\epsilon_{d}$

$$
\tau(R F(d, \epsilon))=d-1 .
$$

Proof: Since $f^{*}$ is surjective and $H^{d-1}(B r(d) ; \pm \mathcal{Z}) \neq 0$, there exists a class $u \in H^{d-1}(S(d) ; \pm \mathcal{Z})$ with $f^{*}(u) \neq 0$. Hence

$$
\operatorname{secat}(\pi)=\operatorname{cat}(f) \geqslant \operatorname{wgt}(u) \geqslant d-1 .
$$

So, for the case of the root finding problem when the degree $d$ is a power of a prime, the best possible solution algorithm requires precisely $d-1$ decisions. Thus, a result far from homotopy theory follows from the homotopical properties of sectional category - a rather surprising consequence.

\subsection{Farber's Topological Complexity}

A mechanical system $\mathcal{S}$ is described by its totality of states $X=X(\mathcal{S})$, this is the configuration space of $\mathcal{S}$. 
Example 58. A planar robot arm with $n$ links has configuration space the $n$-torus $T^{n}$ since the relevant parameters of the system are the $n$ angles between consecutive links.

Example 59. When we have a rigid body in $\mathbb{R}^{3}$, each configuration (relative to a fixed frame) can be identified with an element of the rotation group $\mathrm{SO}(3)$. Thus, $\mathrm{SO}(3)$ is the configuration space for rigid body motion.

Example 60. If $n$ particles (or robots) move in a space $Y$ to avoid collisions, then the configuration space is

$$
F(Y, n)=\left\{\left(y_{1}, \ldots, y_{n}\right) \in Y^{n} ; y_{i} \neq y_{j}, i \neq j\right\}
$$

In particular, if $n$ robots move along a wire network (graph) $\Gamma$ in a factory, then a position for each of the robots gives a point in $F(\Gamma, n)$. The definition of $F(Y, n)$ that we have written above is actually the general topological definition of the configuration space associated to the space $Y$.

In robotics, the fundamental problem is how to control a robot from any one configuration to any other configuration. Now, there are two important requirements.

1) The process of finding the path should work for all pairs of points. In other words, no matter what state the robot is in, there is a control process that can bring it to any given state.

2) The process should be fully automated (i.e., algorithmic). This is particularly important since robots are expected to act without human guidance.

Formally, we write the following.

Problem 61. Let $X$ be the configuration space of a system $\mathcal{S}$. The motion planning problem is to algorithmically determine a continuous path $\gamma: I \rightarrow X$ with $\gamma(0)=$ $A$ and $\gamma(1)=B$ for any $A, B \in X$.

A precise mathematical formulation of the problem is the following (see [5]). Let ev: $X^{I} \rightarrow X \times X$ be the evaluation fibration ev $(\gamma)=(\gamma(0), \gamma(1))$, where $X^{I}$ is the space of all paths $\gamma: I \rightarrow X$. A motion planning algorithm is a continuous section

$$
s: X \times X \rightarrow X^{I}, \quad \text { ev } \circ=1_{X \times X}
$$

Unfortunately, we have the following sobering result. 
Proposition 62. A motion planning algorithm $s: X \times X \rightarrow X^{I}$ exists if and only if $X$ is contractible (i.e., deformable to a point).

Proof: If $X \simeq *$, then there is a homotopy $H: X \times I \rightarrow X$ with $H(x, 0)=x$ and $H(x, 1)=B_{0}$, for fixed $B_{0}$. Given $A, B$, define

$$
\gamma(t)= \begin{cases}H(A, 2 t) & 0 \leqslant t \leqslant 1 / 2 \\ H(B, 2-2 t) & 1 / 2 \leqslant t \leqslant 1\end{cases}
$$

Since this continuously determines a path $\gamma(t)$ for each pair $(A, B)$, we obtain a motion planning algorithm. On the other hand, if a motion planning algorithm $s: X \times X \rightarrow X^{I}$ exists, define $H: X \times I \rightarrow X$ by $H(A, t)=s\left(A, B_{0}\right)(t)$. Then $H(A, 0)=A$ and $H(A, 1)=B_{0}$ because $s$ is a section of ev.

So what can be done for more general spaces if motion planning algorithms only exist for contractible configuration spaces? Well, this is precisely the LS category approach to a space's complexity.

Definition 63. The Topological Complexity of the motion planning algorithm problem for $X$ is

$$
\mathrm{TC}(X)=\operatorname{secat}\left(\mathrm{ev}: X^{I} \rightarrow X \times X\right) .
$$

The idea behind this definition is that we decompose $X \times X$ into open sets $U$ for which there is a motion planning algorithm in $X$. By this we mean that the path that connects two points of $U$ may go outside $U$ itself. The complexity of the configuration space with respect to finding a motion planning algorithm is then measured by the sectional category of the evaluation map ev: $X^{I} \rightarrow X \times X$.

The relation between LS category and topological complexity is expressed by the following inequalities.

Proposition 64. Topological complexity is a homotopy invariant and the following estimates hold

$$
\operatorname{cat}(X) \leqslant \mathrm{TC}(X) \leqslant \operatorname{cat}(X \times X) \leqslant 2 \operatorname{cat}(X) .
$$

For the proof of homotopy invariance, see [5]. The estimate $\mathrm{TC}(X) \leqslant \operatorname{cat}(X \times X)$ is simply (1) of Property 16 while the estimate cat $(X) \leqslant \mathrm{TC}(X)$ follows from (5) of Property 16 when we recognize that the (based) path fibration $P X \rightarrow X$ is a pullback of the evaluation fibration ev: $X^{I} \rightarrow X \times X$ by the mapping $X \rightarrow$ $X \times X, x \mapsto\left(x_{0}, x\right)$ for a fixed basepoint $x_{0} \in X$. Here is the fundamental example (see [5]). 


\section{Proposition 65.}

$$
T C\left(S^{n}\right)= \begin{cases}1 & n \text { odd } \\ 2 & n \text { even }\end{cases}
$$

Proof: Case n odd. Let's break $S^{n} \times S^{n}$ into two open sets.

$$
U=\{(x, y) ; y \neq-x\}, \quad V=\{(x, y) ; y=-x\} .
$$

(Note that the second set is not open, but we can take a small deformable neighborhood around the anti-diagonal to "make it open".) We need to define local sections on these sets. This means specifying a path from $x$ to $y$ in an algorithmic continuous fashion. For $U$, simply take the path that is the unique minimizing geodesic between $x$ and $y$. This makes sense precisely because $y \neq-x$. For $V$ this definition would be a problem since there are two minimizing geodesics. However, here we can use the fact that, since the Euler characteristic of an odd sphere is zero, there is a non-vanishing vector field on $S^{n}$. So, at $x$, take the vector field vector as the initial condition of a geodesic and take this path to $y=-x$. (Here we use the fact that geodesics are great circles.) Since we have local sections on $U$ and $V$, we see that $\operatorname{TC}\left(S^{n}\right) \leqslant 1$. By Proposition 64, we also see that $1=\operatorname{cat}\left(S^{n}\right) \leqslant \mathrm{TC}\left(S^{n}\right) \leqslant 1$, so $\mathrm{TC}\left(S^{n}\right)=1$.

Case $\mathbf{n}$ even. So what is the only difference in the case of $n$ being even? It is simply that the Euler characteristic of an even sphere is 2, not 0 . So we cannot use the method on $V$ to find an algorithmic path. However, we $d o$ know that there is a vector field on $S^{n}$ with only a single zero $x_{0}$, say. So if we define $U$ as above and

$$
V=\left\{(x, y) ; y=-x, x \neq x_{0}\right\}, \quad W=\left\{\left(x_{0},-x_{0}\right)\right\}
$$

then we can use the vector field on $V$ as before. Again, we can take a small contractible neighborhood around $\left(x_{0},-x_{0}\right)$ that deforms to $\left(x_{0},-x_{0}\right)$, so we need only define a path for this single point. For this we can take any path from $x_{0}$ to $-x_{0}$. Therefore, since we cover with three sets $U, V, W, \operatorname{TC}\left(S^{n}\right) \leqslant 2$. To get a good lower bound, we use (3) of Property 16 by considering the element

$$
\begin{aligned}
\chi= & x \otimes 1-1 \otimes x \\
& \in\left(H^{n}\left(S^{n} ; \mathbb{Q}\right) \otimes H^{0}\left(S^{n} ; \mathbb{Q}\right)\right) \oplus\left(H^{0}\left(S^{n} ; \mathbb{Q}\right) \otimes H^{n}\left(S^{n} ; \mathbb{Q}\right)\right) \\
& \cong H^{n}\left(S^{n} \times S^{n} ; \mathbb{Q}\right)
\end{aligned}
$$

where $x \neq 0$ in $H^{n}\left(S^{n} ; \mathbb{Q}\right)$. Now, $\mathrm{ev}^{*}(\chi)=0$ because $X^{I} \simeq X$ and $\mathrm{ev}^{*}(x \otimes 1=$ $x=\mathrm{ev}^{*}(1 \otimes x)$. Also, $\chi^{2} \neq 0$ since graded commutativity of cohomology gives

$$
\chi^{2}=-x \otimes x-(-1)^{n^{2}} x \otimes x
$$


and $n$ is even. (Note that this argument would not work for $n$ odd.) Hence, by (3) of Property 16, TC $\left(S^{n}\right) \geqslant 2$. Thus, $\mathrm{TC}\left(S^{n}\right)=2$.

Remark 66. There are several things to note about this result. First, although TC is a homotopy invariant, we are using geometric entities such as geodesics and vector fields to obtain local sections. More homotopical approaches to TC have more recently appeared that reformulate $\mathrm{TC}$ akin to the reformulation of category in Section 3. Secondly, note that odd spheres realize the lower bound of Proposition 64 while even spheres realize the upper bound.

Here is another case where the lower bound of Proposition 64 is realized. This is an important case because Lie groups are often configuration spaces for systems such as robots.

Theorem 67. If $X$ is a Lie group, then $\mathrm{TC}(X)=\operatorname{cat}(X)$.

Proof: By Proposition 64, we must only show that $\mathrm{TC}(X) \leqslant \operatorname{cat}(X)$. To see this, we use both parts of (5) of Property 16 together with the existence of a multiplication (with inverse and identity $e$ ) on $X$. First, take the pullback

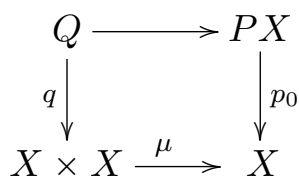

where $\mu: X \times X \rightarrow X$ is given by $\mu(x, y)=y x^{-1}$. The definition of pullback gives $Q=\left\{(x, y, \gamma) \mid \gamma(0)=e, \gamma(1)=y x^{-1}\right\}$. Since we have a pullback, we see that

$$
\operatorname{secat}(q) \leqslant \operatorname{secat}\left(p_{0}\right)=\operatorname{cat}(X) .
$$

But now consider the diagram

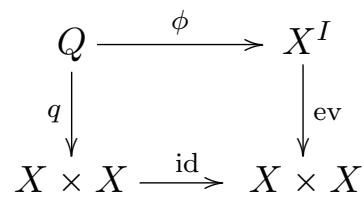

where $\phi(x, y, \gamma)(t)=\gamma(t) \cdot x$. We then have

$$
\mathrm{ev}(\phi(x, y, \gamma))=(\gamma(0) \cdot x, \gamma(1) \cdot x)=(x, y)
$$

so the diagram commutes. Again by (5) of Property 16, we obtain

$$
\mathrm{TC}(X)=\operatorname{secat}(X) \leqslant \operatorname{secat}(q) .
$$

Putting the two inequalities together gives the result. 
Example 68. Recall from Example 58 that the configuration space of a planar robot arm with $n$ links is an $n$-torus $T^{n}$. We immediately see that $\mathrm{TC}\left(T^{n}\right)=n$ since we know cat $\left(T^{n}\right)=n$. So a solution to the motion planning problem requires a covering of $T^{n} \times T^{n}$ by at least $n+1$ open sets over which local sections (i.e., motion plans) can be constructed.

Example 69. Recall from Example 59 that $\mathrm{SO}(3)$ is the configuration space for rigid body motion. By the above, we have that $\mathrm{TC}(\mathrm{SO}(3))=3$ since it is known that $\mathrm{SO}(3) \cong \mathbb{R} \mathrm{P}^{3}$ and we have seen in Theorem 3 that $\operatorname{cat}\left(\mathbb{R P}^{3}\right)=3$. This means that if we have a robot arm with a hand giving it orientation, then there are obstructions to passing continuously from any state to any other state. We require four open sets in which motion planning can be accomplished. This tells engineers that they should expect discontinuous motions at times and that some type of intervention might be necessary to avoid breakage of the robot arm.

Here is a result for configuration spaces on $\mathbb{R}^{m}[6]$.

\section{Theorem 70.}

$$
\mathrm{TC}\left(F\left(\mathbb{R}^{m}, n\right)\right)= \begin{cases}2 n-2 & m \text { odd } \\ 2 n-3 & m \text { even }\end{cases}
$$

Now, TC does not just have applications to robotics. There is a feedback loop to topology as well. A longstanding problem in topology has been the question of the smallest dimensional Euclidean space that supports an immersion of a given real projective space. Here is a very surprising result that provides a new approach to the problem [5].

Theorem 71. For $n \neq 1,3,7, \mathrm{TC}\left(\mathbb{R P}^{n}\right)$ is the smallest $k$ such that $\mathbb{R P}^{n}$ admits an immersion into $\mathbb{R}^{k}$.

Remark 72. Note that Example 69 gave $\mathrm{TC}\left(\mathbb{R P}^{3}\right)=3$, but $\mathbb{R P}^{3}$ does not immerse in $\mathbb{R}^{3}$. Thus, the exceptional cases arise when $\mathbb{R}^{k}, k=2,4,8$ have "product” structures (complex, quaternion, Cayley numbers). Note also that, while Proposition 64 tells us that $2 \leqslant \mathrm{TC}\left(\mathbb{R P}^{2}\right) \leqslant 4$, Theorem 71 gives $\mathrm{TC}\left(\mathbb{R P}^{2}\right)=3$ since $\mathbb{R} \mathrm{P}^{2}$ immerses in $\mathbb{R}^{3}$ (as Boy's surface say) but not in $\mathbb{R}^{2}$.

Proposition 65 begs the question of determining spaces with low topological complexity. In [10], these spaces were identified.

Theorem 73 (Grant-Lupton-Oprea). If $\mathrm{TC}(X)=1$, then $X$ is homotopy equivalent to some sphere of odd dimension. Moreover, if $X$ is also a closed manifold, then $X$ is homeomorphic to an odd sphere. 
This result has a rather complicated proof, but the starting point is the recognition that the inequality cat $(X) \leqslant \mathrm{TC}(X)=1$ implies that $X$ is a co-H-space (i.e., a space with co-multiplication) since $\operatorname{cat}(X)=1$ identifies these spaces. Now, there are co-H-spaces that are not spheres. In fact, every suspension $\Sigma X$ is a co-H-space and cat $(\Sigma X)=1$ as can be seen by decomposing $\Sigma X$ into its top and bottom cones. So Theorem 73 is giving yet another indication that TC is a more powerful invariant than LS category.

A major question in the subject is, what is the topological complexity of a $K(\pi, 1)$. Eilenberg and Ganea showed that, when the (geometric) dimension of a $K(\pi, 1)$ is greater than 3 , then

$$
\operatorname{cd}(\pi)=\operatorname{cat}(K(\pi, 1))=\operatorname{dim}(K(\pi, 1)) .
$$

Here, $\operatorname{cd}(\pi)$ is the cohomological dimension of the group $\pi$, so we only get a finite number above when $\operatorname{cd}(\pi)<\infty$ : in particular, only when $\pi$ is torsionfree. There are some determinations of TC for $K(\pi, 1)$ which often use some type of auxiliary structure associated to the group $\pi$ that enables an application of Property 16 - 3 (e.g. see [3]). Here is a more general result that centers on the subgroup structure of $\pi$ [11].

Theorem 74 (Grant-Lupton-Oprea). If $A$ and $B$ are complementary subgroups of $\pi$ (i.e., $A B=\pi$ and $A \cap B=\varnothing$ ), then

$$
\operatorname{cd}(A \times B) \leqslant \mathrm{TC}(K(\pi, 1)) .
$$

Using this result, we can recover lower bounds for TC for various types of $K(\pi, 1)$ 's such as right-angled Artin groups and braid groups. Furthermore, using much harder arguments, we obtain the following result.

Corollary 75. Let $\mathcal{H}$ denote the Higman group with presentation

$$
\left\langle x, y, z, w \mid x y x^{-1} y^{-2}, y z y^{-1} z^{-2}, z w z^{-1} w^{-2}, w x w^{-1} x^{-2}\right\rangle .
$$

Then $\operatorname{TC}(\mathcal{H})=4$.

It is known that the group $\mathcal{H}$ is acyclic (it has the same integer homology as a trivial group), and so $H^{*}(\mathcal{H} ; k)=0$ (in positive degrees) for every abelian group $k$. Moreover, $\mathcal{H}$ has no non-trivial finite quotients, so it has no non-trivial finite dimensional representations over any field. It then follows that if $M$ is any coefficient $\mathcal{Z}[\mathcal{H}]$-module which is finitely generated as an abelian group, then $H^{*}(\mathcal{H} ; M)=0$. Thus the group $\mathcal{H}$ is difficult to distinguish from a trivial group using cohomological invariants. 
On the other hand, since $\mathcal{H}$ is not a free group we have $\operatorname{cd}(\mathcal{H}) \geqslant 2$. The twodimensional complex associated to the presentation $P$ is known to be aspherical and it follows that $\operatorname{cat}(\mathcal{H})=\operatorname{cd}(\mathcal{H})=2$. Thus the topological complexity of Higman's group satisfies $2 \leqslant \mathrm{TC}(\mathcal{H}) \leqslant 4$. A nontrivial argument using BassSerre theory shows the result that $\mathrm{TC}(\mathcal{H})=4$.

\section{Final Words}

We have seen several themes throughout this work. First, LS category and its offspring often serve as intermediaries or translators between the worlds of geometry or applied mathematics and topology, providing measures of complexity estimable by algebraic means. Secondly, the ubiquity of $K(\pi, 1)$-manifolds or manifolds such as $\mathbb{R P}^{n}$ where cat $=$ dim in applications shows that the power of LS category-like invariants is perhaps felt the most when the fundamental group is present. Finally, the emergence of TC as a new player in applied topology gives credence to the belief that LS category is a living breathing subject as we approach the middle of the second decade of the 21 st century.

\section{Acknowledgements}

I would like to thank the organizers of the Varna conference on Geometry, Quantization and Integrability, Ivaïlo Mladenov and Mariana Hadzhilazova, for the invitation to give the set of lectures from which this paper is derived as well as for their hospitality during my stay.

Also, it is a pleasure to acknowledge that this work was partially supported by a grant from the Simons Foundation (\#244393 to John Oprea).

\section{References}

[1] Arnold V., Mathematical Methods of Classical Mechanics, Translated from the 1974 Russian original by K. Vogtmann and A. Weinstein, $2^{\text {nd }}$ Edn, Graduate Texts in Mathematics 60, Springer, Berlin 1989.

[2] Cheeger J. and Gromoll D., The Splitting Theorem for Manifolds of NonNegative Ricci Curvature, J. Diff. Geom. 6 (1971) 119-128.

[3] Cohen D. and Pruidze G., Motion Planning in Tori, Bull. London Math. Soc. 40 (2008) 249-262. 
[4] Cornea O., Lupton G., Oprea J. and Tanré D., Lusternik-Schnirelmann Category, Surveys and Monographs 103, AMS, Providence 2003.

[5] Farber M., Invitation to Topological Robotics, Zurich Lectures in Advanced Mathematics, European Mathematical Society, Zurich 2008, see the review in this journal at http://www.bio21.bas.bg/jgsp/jgsp_files/ vol16/Farber.pdf

[6] Farber M. and Grant M., Topological Complexity of Configuration Spaces, Proc. Amer. Math. Soc. 137 (2009) 1841-1847.

[7] Farber M., Grant M. and Yuzvinsky S., Topological Complexity of Collision Free Motion Planning Algorithms in the Presence of Multiple Moving Obstacles, In: Topology and Robotics, Contemp. Math. 438, AMS, Providence 2007, pp 75-83.

[8] Fukaya K. and Ono K., Arnold Conjecture and Gromov-Witten Invariant, Topology 38 (1999) 933-1048.

[9] Félix Y., Oprea J. and Tanré D., Algebraic Models in Geometry, Oxford Graduate Texts in Mathematics 17, Oxford University Press, Oxford 2008.

[10] Grant M., Lupton G. and Oprea J., Spaces of Topological Complexity One, Homology Homotopy Appl. 15 (2013) 73-81.

[11] Grant M., Lupton G. and Oprea J., New Lower Bounds for the Topological Complexity of Aspherical Spaces, arXiv:1309.4192 [math.AT].

[12] Hofer H. and Zehnder E., Symplectic Invariants and Hamiltonian Dynamics, Reprint of the 1994 edition, Modern Birkhäuser Classics, Birkhäuser, Basel 2011.

[13] Liu G. and Tian G., Floer Homology and Arnold Conjecture, J. Differential Geom. 49 (1998) 1-74.

[14] Musin O., Borsuk-Ulam Type Theorems for Manifolds, Proc. Amer. Math. Soc. 140 (2012) 2551-2560.

[15] Nyman K. and Su F., A Borsuk-Ulam Equivalent That Directly Implies Sperner's Lemma, Amer. Math. Monthly 120 (2013) 346-354.

[16] Oprea J., Category Bounds for Nonnegative Ricci Curvature Manifolds with Infinite Fundamental Group, Proc. Amer. Math. Soc. 130 (2002) 833-839.

[17] Kapovitch V., Petrunin A. and Tuschmann W., Nilpotency, Almost Nonnegative Curvature, and the Gradient Flow on Alexandrov Spaces, Ann. of Math. 171 (2010) 343-373.

[18] Oprea J. and Strom J., Mixing Categories, Proc. Amer. Math. Soc. 139 (2011) 3383-3392. 
[19] Oprea J. and Strom J., On Fox's M-Dimensional Category and Theorems of Bochner Type, Topology Appl. 159 (2012) 1448-1461.

[20] Petersen P., Riemannian Geometry, Graduate Texts in Mathematics 171, Springer, New York 1998.

[21] Rudyak Y. and Oprea J., On the Lusternik-Schnirelmann Category of Symplectic Manifolds and the Arnold Conjecture, Math. Z. 230 (1999) 673-678.

[22] Rudyak Y., On Category Weight and Its Applications, Topology 38 (1999) 37-55.

[23] Rudyak Y., On Strict Category Weight, Gradient-Like Flows, and the Arnold Conjecture, Internat. Math. Res. Notices 5 (2000) 271-279.

[24] Smale S., On the Topology of Algorithms, I. J. Complexity 3 (1987) 8189.

[25] Strom J., Essential Category Weight and Phantom Maps, In: Cohomological Methods in Homotopy Theory (Bellaterra, 1998), Progr. Math. 196, Birkhäuser, Basel 2001, pp 409-415.

[26] Su F., Borsuk-Ulam Implies Brouwer: A Direct Construction, Amer. Math. Monthly 104 (1997) 855-859.

[27] Tralle A. and Oprea J., Symplectic Manifolds with No Kähler Structure, Lecture Notes in Mathematics 1661, Springer, Berlin 1997.

[28] Vassiliev V., Complements of Discriminants of Smooth Maps: Topology and Applications, Revised Edition, Translations of Mathematical Monographs 98, AMS, Providence 1992.

[29] Yamaguchi T., Collapsing and Pinching Under a Lower Curvature Bound, Ann. Math. 133 (1991) 317-357.

[30] Yano K. and Bochner S., Curvature and Betti Numbers, Annals of Mathematics Studies 32, Princeton University Press, Princeton 1953.

John Oprea Department of Mathematics

Cleveland State University

Cleveland $\mathrm{OH}$

44115 USA

E-mail address: j.oprea@csuohio.edu 